Feature article

\title{
Combining theory and experiment for X-ray absorption spectroscopy and resonant X-ray scattering characterization of polymers
}

\author{
Gregory M. Su a, Isvar A. Cordova a, Michael A. Brady ${ }^{\mathrm{a}, \mathrm{b}}$, David Prendergast ${ }^{\mathrm{b}}$, \\ Cheng Wang ${ }^{\text {a, * }}$ \\ a Advanced Light Source, Lawrence Berkeley National Laboratory, Berkeley, CA 94720, USA \\ ${ }^{\mathrm{b}}$ The Molecular Foundry, Lawrence Berkeley National Laboratory, Berkeley, CA 94720, USA
}

\section{A R T I C L E I N F O}

\section{Article history:}

Received 23 May 2016

Accepted 28 June 2016

Available online 4 July 2016

\section{Keywords:}

Polymer

Resonant scattering

Resonant reflectivity

NEXAFS

XPCS

Simulations

In situ

Operando

\begin{abstract}
A B S T R A C T
An improved understanding of fundamental chemistry, electronic structure, morphology, and dynamics in polymers and soft materials requires advanced characterization techniques that are amenable to in situ and operando studies. Soft X-ray methods are especially useful in their ability to non-destructively provide information on specific materials or chemical moieties. Analysis of these experiments, which can be very dependent on X-ray energy and polarization, can quickly become complex. Complementary modeling and predictive capabilities are required to properly probe these critical features. Here, we present relevant background on this emerging suite of techniques. We focus on how the combination of theory and experiment has been applied and can be further developed to drive our understanding of how these methods probe relevant chemistry, structure, and dynamics in soft materials.
\end{abstract}

Published by Elsevier Ltd.

\section{Contents}

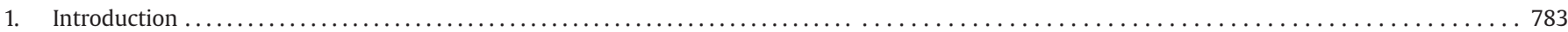

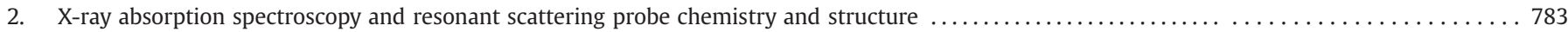

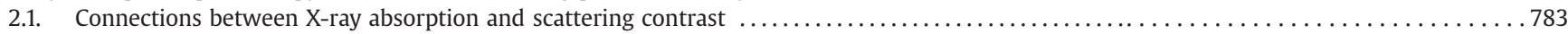

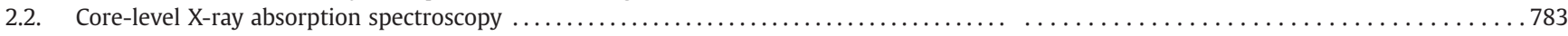

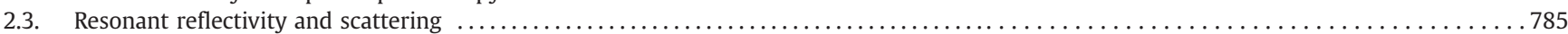

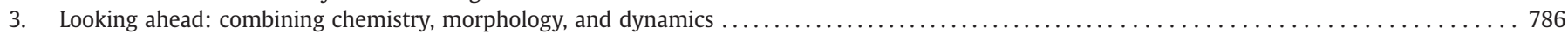

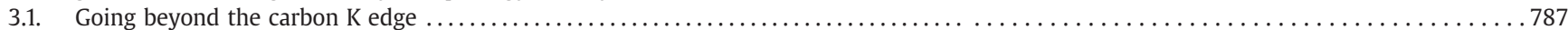

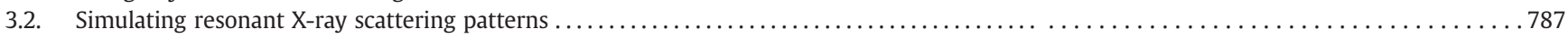

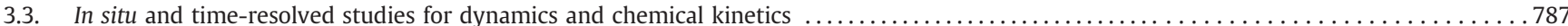

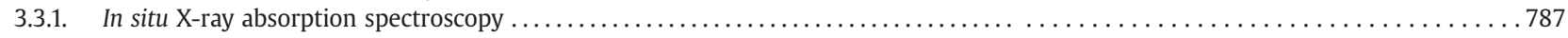

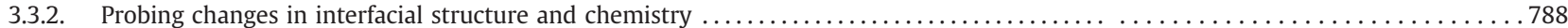

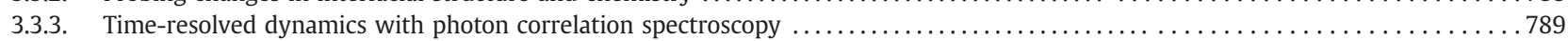

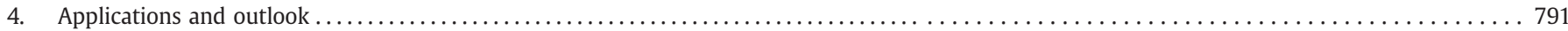

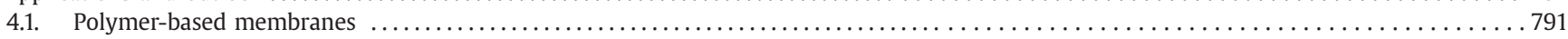

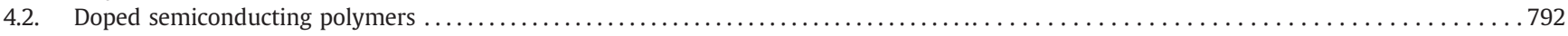

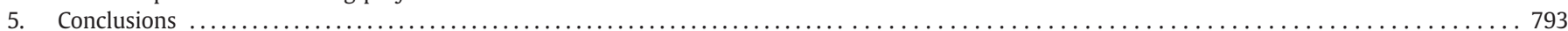

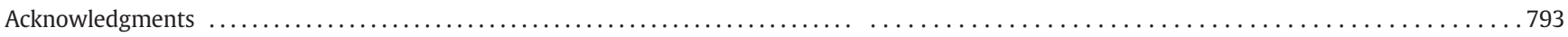

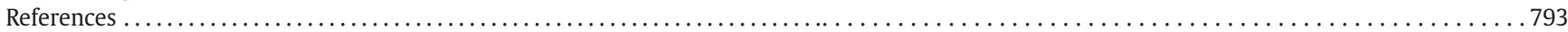

\footnotetext{
* Corresponding author.

E-mail addresses: gsu@lbl.gov (G.M. Su), iacordova@lbl.gov (I.A. Cordova), mabrady@lbl.gov (M.A. Brady), dgprendergast@lbl.gov (D. Prendergast), cwang2@ lbl.gov (C. Wang).
}

http://dx.doi.org/10.1016/j.polymer.2016.06.068 0032-3861/Published by Elsevier Ltd.

(C) 2016. This manuscript version is made available under the Elsevier user license http://www.elsevier.com/open-access/userlicense/1.0/ 


\section{Introduction}

The intricate connections among chemical structure, local intermolecular and global morphology, and kinetics in polymeric materials determine the fundamental properties of many polymerbased applications. These include flexible electronics [1-4], gas separations [5-7], polymer electrolytes for batteries, fuel cells, and water desalination [8-10], and lithographic patterning [11-13] just to name a few. It has remained challenging to fully understand the relationships between chemistry and structure, and the growing demand to characterize polymers under in situ or operando conditions relevant to a specific application and as a function of time creates additional challenges. Probing detailed information such as this requires advanced characterization methods that need to be complemented by theory. Energy-tunable soft X-rays are the basis for a unique set of tools that are sensitive to molecular and electronic structure, spatial and orientation information, and timeresolved dynamics.

Soft X-rays span an energy range that includes the core-level 1s electronic transitions (K edges) of some of the most common elements found in polymers, for example, carbon, nitrogen, and oxygen. Soft X-ray spectroscopy has an inherent ability to not only selectively probe for the presence of these elements, but also be sensitive to various bonding environments, functional groups, and the orientation of chemical moieties. In addition to the chemistry, soft X-rays can be used for energy-dependent scattering experiments that probe spatial information and characteristic length scales that range from a few nanometers to hundreds of nanometers. The interdependence of spectroscopy and scattering allows for unique capabilities to understand the length scales associated with certain components, moieties or molecular orientations. However, understanding the relationships that connect molecular structure, spectroscopy, and scattering can be very complex, and complementary simulations are needed to build fundamental knowledge and help interpret experiments. An improved ability to leverage theoretical predictions will enable soft X-ray methods to move forward into the realm of in situ characterization and bridge the gaps among chemistry, morphology, and dynamics in polymers and soft materials.

\section{X-ray absorption spectroscopy and resonant scattering probe chemistry and structure}

\subsection{Connections between X-ray absorption and scattering contrast}

Near edge X-ray absorption fine structure (NEXAFS) spectroscopy and resonant soft X-ray scattering (RSoXS) and reflectivity (RSoXR) are naturally complementary techniques, and information regarding a materials absorption properties is needed to understand RSoXS and RSoXR. Unlike hard X-ray scattering or electron microscopy where contrast arises due to differences in electron density, at energies near an absorption edge the complex index of refraction, $n(E)=1-\delta(E)+i \beta(E)$, and hence scattering contrast, varies significantly. NEXAFS data provides information related to $\beta$, and this can be extended in energy range and scaled based on a material's composition using the Henke atomic scattering factor database [14]. The real part of the index of refraction, $\delta$, can be calculated from $\beta$ through a Kramers-Kronig integral relation and the resulting contrast between different phases, $\Delta \delta^{2}+\Delta \beta^{2}$, determined as a function of energy. This procedure is often done for organic systems, and an example is shown in Fig. 1. It is clear that at energies near an absorption edge, scattering contrast even between materials with similar electron densities can be greatly enhanced. This is one of the main advantages that soft X-rays have for characterizing polymers. However, the detailed connections between a a)

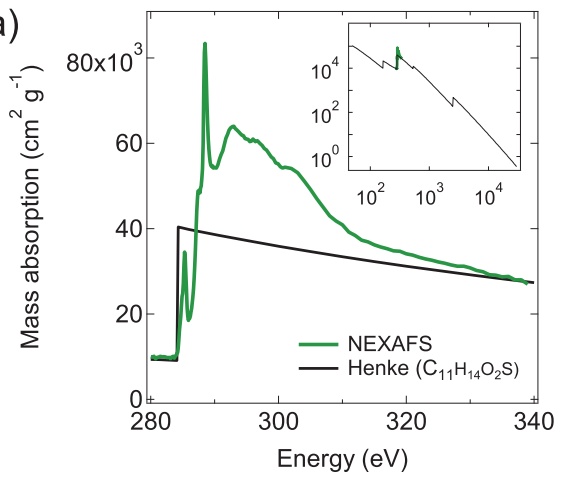

b)

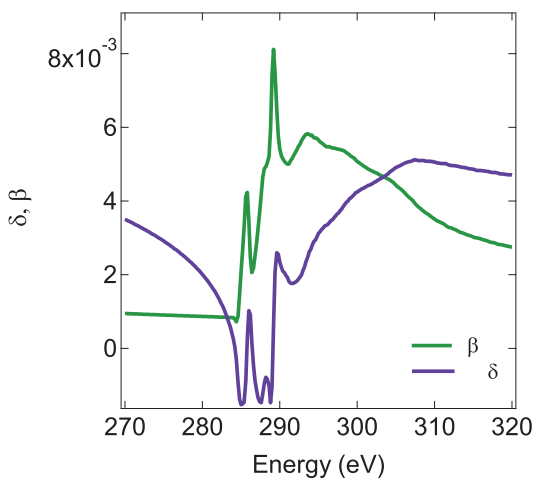

Fig. 1. Measured NEXAFS data can be scaled and extended with the Henke database, as shown in (a). The inset shows a log-log plot to demonstrate how the Henke database is used to approximate values at energies outside of where NEXAFS data was collected. The real part of the complex index of refraction, $\delta$, determined through a KramersKronig relation, is plotted with the imaginary part, $\beta$, in (b). The example here is for a polythiophene, poly[3-(ethyl-5-pentanoate)thiophene-2,5-diyl]

materials absorption properties and resonant scattering can make it challenging to discern features such as contributions from specific chemical moieties or orientation effects. This is an area where progress in effective combination of theoretical predictions and experiments is needed to improve advanced characterization.

\subsection{Core-level X-ray absorption spectroscopy}

Fundamental knowledge of NEXAFS is a prerequisite to also understanding RSoXS and RSoXR. The subtleties in scattering contrast between polymers stems from the unique absorption profiles of each material. Since NEXAFS is sensitive to specific functional groups and bonds, materials that are composed of the same elements, for example polystyrene and polyethylene which both contain just carbon and hydrogen, can have very distinct NEXAFS profiles. NEXAFS is itself a useful tool in polymer science, and has been widely used to examine thin film properties such as surface composition [15-18] and molecular orientation in ordered polymers [19-23]. Furthermore, absorption differences between polymers can be utilized for chemical sensitivity in real space imaging of thin films via scanning transmission X-ray microscopy (STXM) [24-31].

NEXAFS spectra can be difficult to understand in detail, and complementary simulations are especially useful to help discern features such as contributions from specific atoms to certain peaks or intensity changes due to X-ray polarization and molecular orientation. Many recent studies utilize density functional theory (DFT) $[32,33]$ for predicting core-level transitions. Although DFT is limited to ground state properties and reliable predictions of excited states are more difficult [34], it has proven successful for simulations of X-ray absorption spectroscopy (XAS) [35,36]. The 
advantages of combining NEXAFS calculations with experiment have already shown success for polymer systems. Simulations based on a single polymer repeat unit can be helpful in deconvoluting overall NEXAFS spectra into contributions from atoms at unique sites, allowing for a better understanding of features such as relative peak intensities and origins of peak splitting, as shown in Fig. 2a [37,38]. Calculations can aid in determining the overall and intrachain orientation in polymers. For instance, chains in rubaligned poly(tetrafluoroethylene) (PTFE) on gold tend to adopt a helical over a zig-zag conformation, and this is determined by comparing experimental results to simulations of model oligomers in both geometries [39]. However, the chain conformation of PTFE may vary depending on specific sample preparation conditions. Calculations that take into account different electric field polarization directions can reveal overall chain axis orientation, for example, along the rubbing direction [40]. NEXAFS is sensitive not only to structure and orientation, but also to changes in chemistry. Chemical reactions, such as site specific bond scission and ionic fragmentation, can occur as a result of core excitation [41]. This allows NEXAFS to inherently probe soft X-ray induced chemical reactions in situ, and this has been demonstrated for poly(methyl methacrylate) (PMMA) and poly-(isopropenyl acetate) (PiPAc) a)

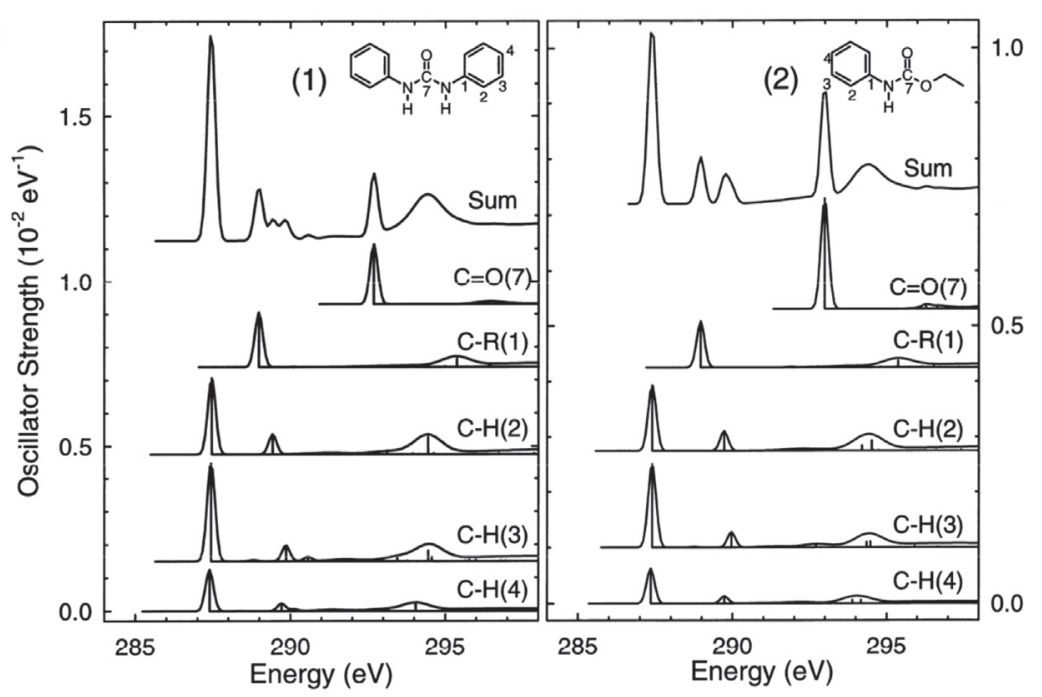

b)

d)

c)

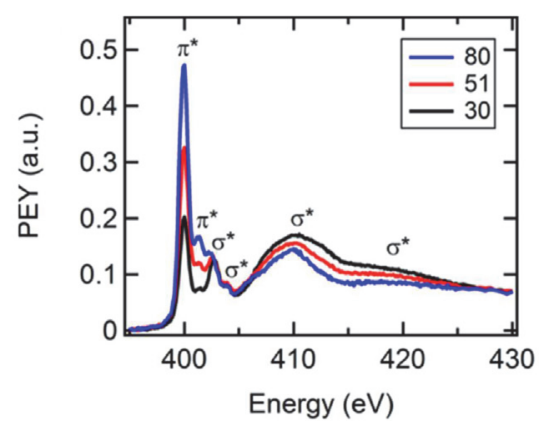

.0 .0

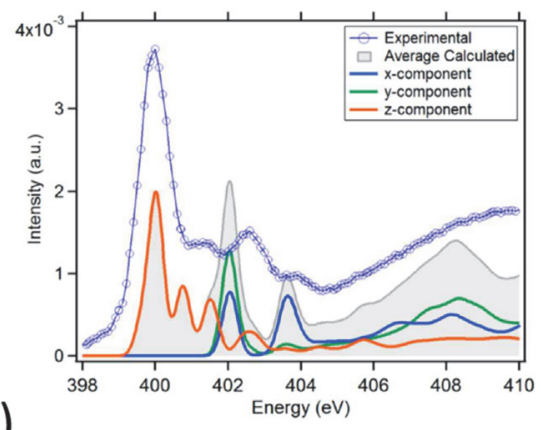

)

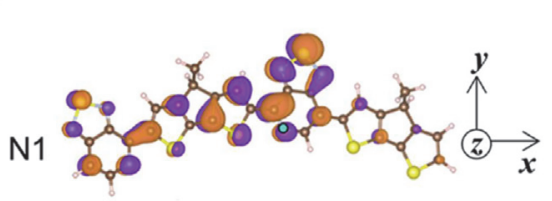

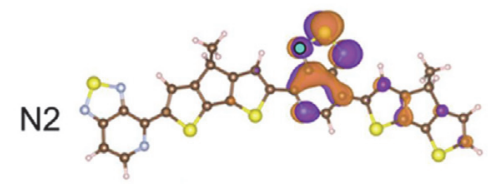

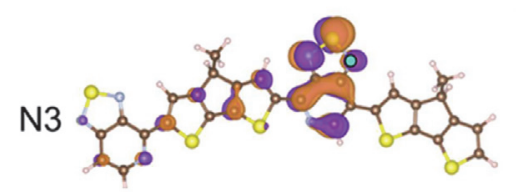

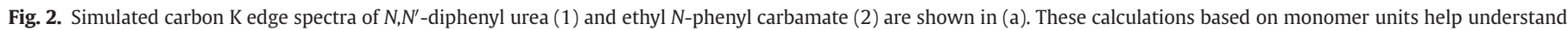

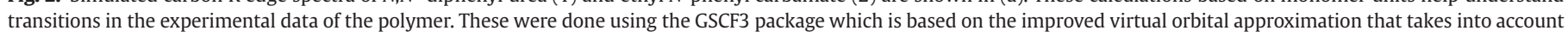

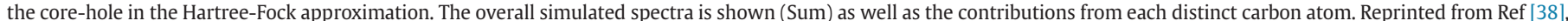

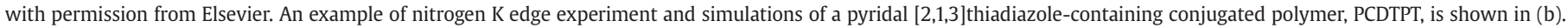

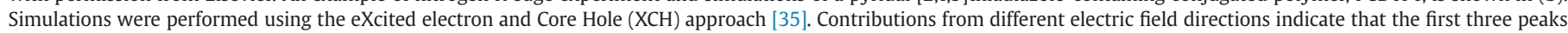

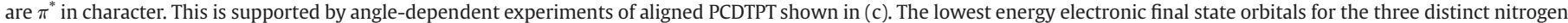

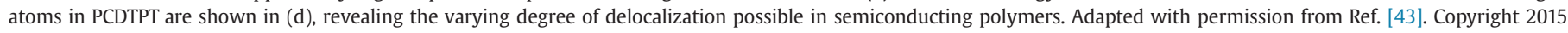
American Chemical Society. 
where a combination of experiment, DFT calculations and molecular dynamics help understand scission of the $\mathrm{C}-\mathrm{O}$ bond and its relation to potential energy surfaces and bond elongation upon $\mathrm{X}$ ray absorption [42].

Complementary NEXAFS predictions are also beneficial for functional polymers such as semiconducting [44] and ion conducting polymers. In semiconducting polymers, electronic structure and molecular orientation are especially important for charge transport and NEXAFS can provide information on these properties. Additional complications need to be considered since the electronic wavefunctions in conjugated polymers are often delocalized over many repeat units [45]. Interpreting the NEXAFS profiles of complex molecular species is often based on the building block model, where a compound is broken down into local subunits (diatomic at the simplest level) to interpret overall spectra [15,46,47]. However, NEXAFS spectra of complex molecular species and polymers are often more complicated than the building block model predicts [48], especially in materials like semiconducting polymers with their delocalized $\pi$ electrons [49,50]. This highlights the need for X-ray absorption spectroscopy simulations for these materials. Only limited work has been done to predict NEXAFS of semiconducting polymers, but these studies demonstrate its potential and utility. For example, simulations have been able to help assign core-level transitions needed for orientation analysis [43,20] (Fig. 2), and help make connections between excited state delocalization and the formation of free charges versus excitons [51]. The combination of theory and experiment for X-ray absorption spectroscopy in functional polymers is still in its early stages, and opportunities exist to improve fundamental understanding of how factors such as delocalized wavefunctions, charge transfer, and backbone geometry influence NEXAFS spectra. Examples of polymer-based applications that can take advantage of soft X-ray probes are presented in Section 4.

\subsection{Resonant reflectivity and scattering}

RSoXS and RSoXR take advantage of the unique core-level absorption profiles of polymers to provide statistically averaged characteristic length scales related to many factors including, but not limited to, phase separation or molecular orientation [52]. As discussed in section 2.1, there are important connections between the real $(\delta)$ and imaginary $(\beta)$ parts of the complex index of refraction that need to be understood. At this fundamental level, RSoXR can be combined with NEXAFS, atomic scattering factors from the Henke database, and the Kramers-Kronig relation to accurately determine $\delta$ and the index of refraction of a polymer in a self-consistent manner without the need for separate mass thickness measurements [53]. The unique features in the index of refraction can be leveraged in RSoXR to gain chemical specificity by tuning the incident X-ray energy and probe orientation or composition through the depth of thin film. For example, RSoXR can characterize roughness or interfacial width of buried polymer interfaces in bilayer or block copolymer systems [54-56], which would be difficult to probe with hard X-ray reflectivity, and likely require chemical modification through deuteration for neutron based experiments. RSoXR is able to assist in characterizing phase separated block copolymers to, for example, help distinguish between vertical and parallel lamellae [57].

Many polymers, including conjugated or liquid crystalline polymers, have asymmetric properties and absorption peaks that correspond to transition dipole moments with well-defined directions that are localized to specific chemical moieties. The control of X-ray polarization that undulator insertion devices provide allows RSoXR an opportunity to probe molecular orientation through the depth of a film, which has been shown for polymers [58] and organic ionic liquids [59]. However, these properties also make it more difficult to properly determine the optical constants from NEXAFS data, and X-ray reflectivity can prove useful as a reference and comparison for $a b$ initio calculations of the dielectric tensor [58]. This has also been demonstrated for a self-assembled monolayer of 1,4-benzenedimethanethiol on gold, where the film's anisotropic dielectric constants were determined based on $a b$ initio DFT simulations of the polarization dependent absorption cross section. Structural parameters such as the molecular tilt angle, monolayer thickness, and packing structure were determined by fitting to experimental RSoXR [60], as shown in Fig. 3a and b.

Complementary to reflectivity, RSoXS has recently emerged as an effective characterization tool for a range of soft materials, including polymeric systems [21,52,61-67]. RSoXS has seen popular use for thin films of conjugated polymer and complex organic semiconductor blends [68-72]. RSoXS has the ability to interrogate in-plane and out-of-plane structure since information from multiple scattering angles can be collected simultaneously on a twodimensional detector and experiments can be done in transmission and grazing incidence geometries. Similar to RSoXR, RSoXS takes advantage of the energy and orientation dependent complex index of refraction near absorption edges to achieve high contrast and chemical sensitivity. Consequently, it is important to understand the complex index of refraction of the materials of interest, at least based on NEXAFS measurements. Even a basic understanding of the absorption differences among individual polymers or components in a blend is sufficient to distinguish differences in characteristic length scales, such as the core vs. outer layer in structured polymer nanoparticles [73] and distinct spacing of various phases in a cylinder forming triblock copolymer [64] or a mesoporous block copolymer film [74].

The transmission geometry and multiple scattering angles detectable in an RSoXS experiment enable unique aspects of molecular orientation to be probed. This allows RSoXS to go beyond RSoXR and investigate in-plane orientation with polarized incident $\mathrm{X}$-rays. A unique feature in polarized scattering that is often seen, especially in semicrystalline polymers, is anisotropic twodimensional scattering (varying intensity as a function of azimuthal angle for a given $Q$ ), which has been attributed to molecular alignment at domain interfaces or fibrillar bulk ordering [75]. This anisotropic scattering occurs in samples that are globally isotropic in-plane and the direction of scattering intensity anisotropy changes with incident $\mathrm{X}$-ray polarization direction, suggesting this feature arises due to core-level transitions with specific dipole moment directions and local molecular arrangement. Several cases of scattering anisotropy have been observed in blends of conjugated polymers and polymer-fullerene blends [68,70-72,75,76], but it can also be observed in a single semicrystalline polymer, as shown in Fig. 3. Depolarized diffuse scattering at large scattering vectors and energies near absorption edges reveals short-range orientation fluctuations of functional groups, as shown for atactic polystyrene [77]. Anisotropic scattering patterns can reveal orientation in liquid crystalline materials, for example, the half-pitch in a bent core liquid crystal that forms a twisted smectic layer structure [78]. Furthermore, resonant scattering can uncover the spatial periodicity in a twist-bend liquid crystal phase that has no modulation in electron density [79].

A full understanding of the origins of this phenomenon is still underway [75]. Challenges exist due to the many details present, ranging from an understanding of the core-level transitions and orientation dependence on the level of individual atoms and functional groups, to intermolecular interactions and local and global phase separation and structure. A complicated problem such as this highlights the need for theoretical developments to help understand subtle connections between spectroscopy and scattering, and push the capabilities of soft X-ray characterization forward. 
a)
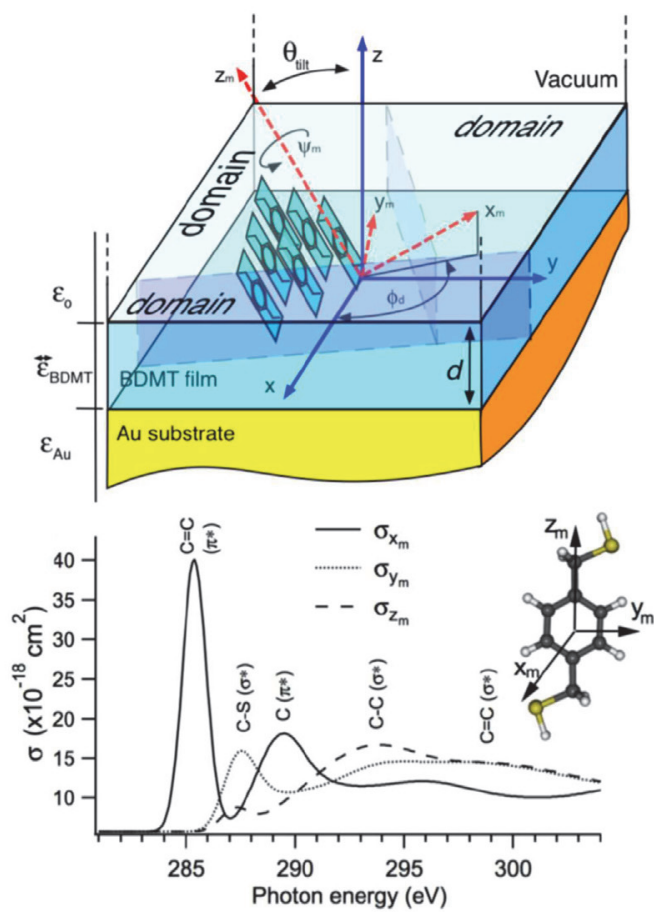

b)
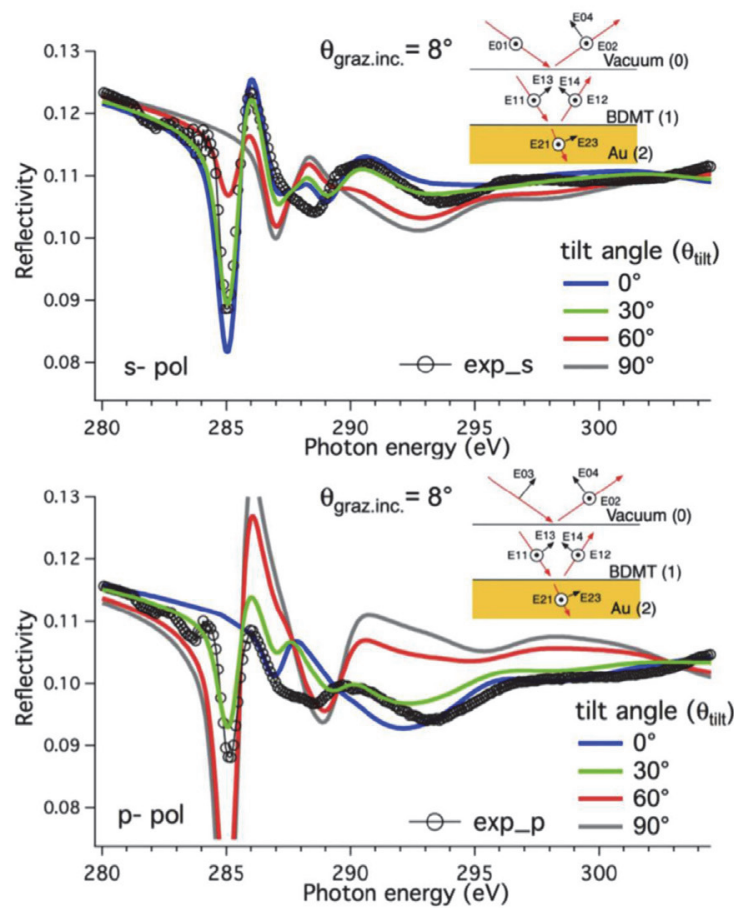

c)

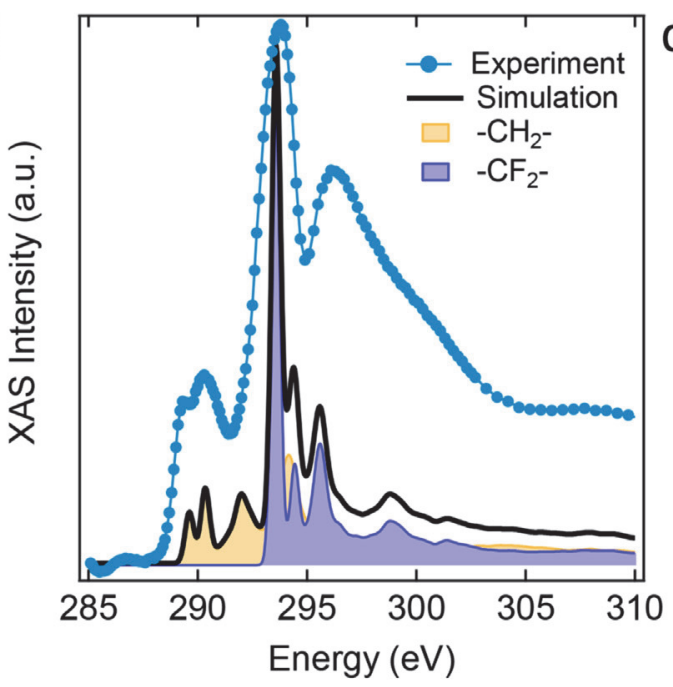

d)

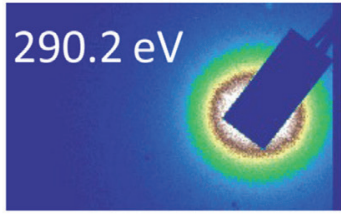

e)
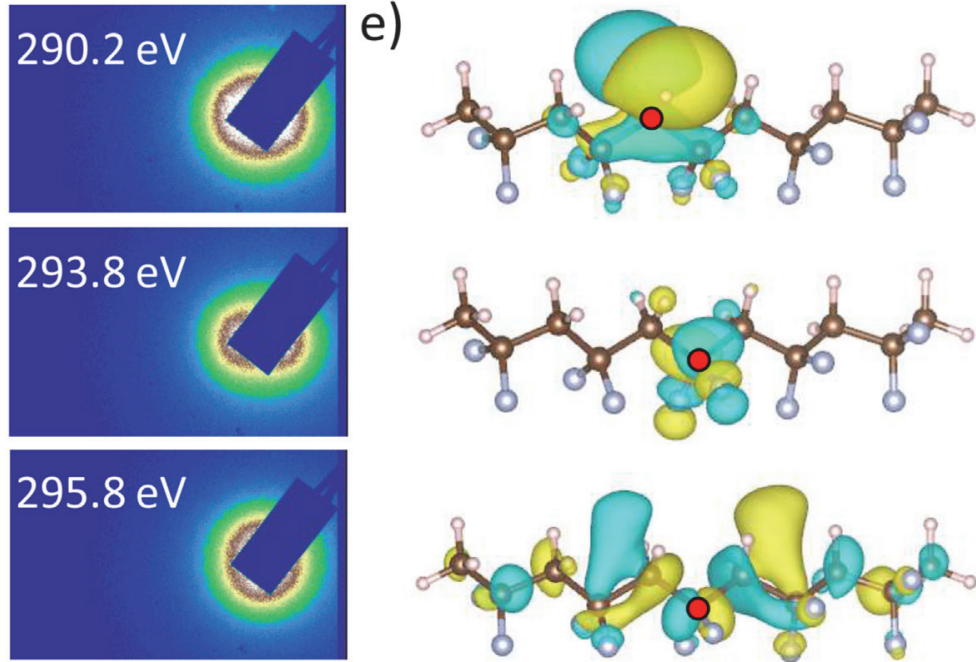
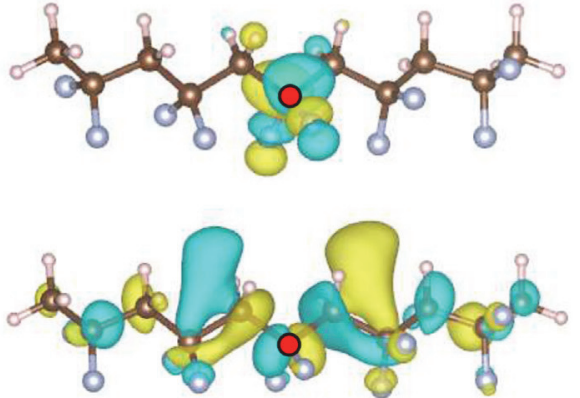

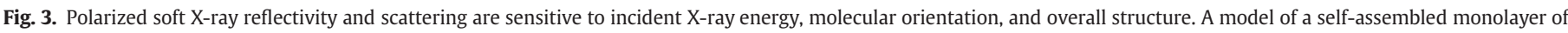

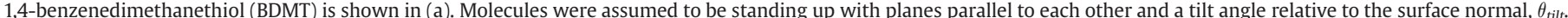

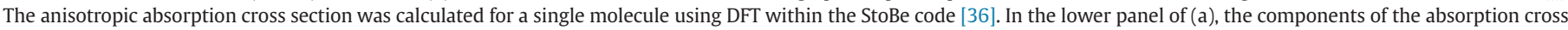

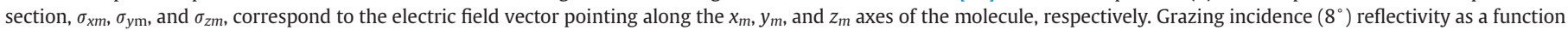

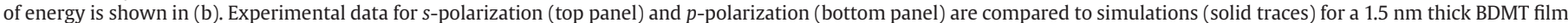

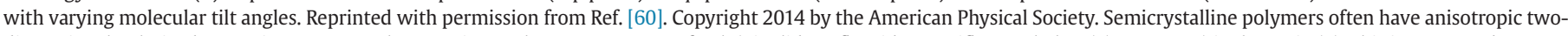

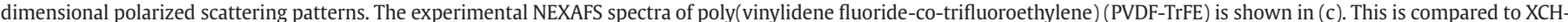

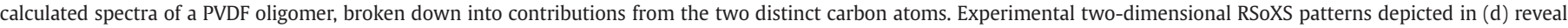

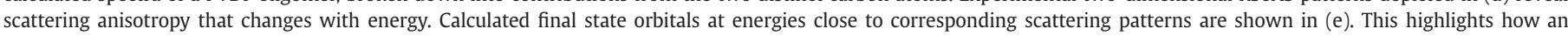
understanding of NEXAFS transitions and transitions dipole moments are connected to polarized RSoXS.

\section{Looking ahead: combining chemistry, morphology, and dynamics}

The combination of X-ray absorption spectroscopy with resonant reflectivity and scattering undoubtedly can reveal critical information about chemistry and morphology in polymers and soft matter, not only in the bulk, but also in thin films and at interfaces. There is a need to understand this information in situ during relevant operating conditions and as a function of time to elucidate structural dynamics and chemical kinetics. This will require even more insights from theory in order to go beyond modeling results after data collection to predicting spectroscopy and scattering $a$ priori. 


\subsection{Going beyond the carbon $K$ edge}

The majority of work done on XAS and RSoXS of polymers focuses on the carbon $\mathrm{K}$ edge since carbon is the most prevalent atomic species in these materials. There are often other elements present in polymers, for example, nitrogen, oxygen, fluorine, and sulfur, that can provide very useful complementary information. Moreover, since there tends to be fewer of these elements in distinct bonding environments compared to carbon, theoretical modeling and experimental data analysis can be more straightforward. For example, the nitrogen $\mathrm{K}$ edge can be used to quantify orientation in a planar conjugated polymer [43], as shown in Fig. 2. In this case, nitrogen atoms are only present on the $[1,2,5]$ thiadiazole[3,4-c]pyridine moiety in the backbone making NEXAFS simulations, peak assignment and orientation analysis less complicated.

In addition to elements with $1 \mathrm{~s}$ absorption edges in the soft Xray regime, many polymers contain elements with higher energy absorption K edges, for example phosphorus (2.145 keV) and sulfur (2.472 keV). Even though these $\mathrm{K}$ edges start to go beyond the energy range of many soft X-ray beamlines, there is much interest to increase capabilities in this "tender" $\mathrm{X}$-ray (2 keV-7 keV) regime. Although typically less common due to the limited number of endstations that can perform XAS or RSoXS near the sulfur and phosphorus $\mathrm{K}$ edges, notable work has been done on sulfur edge XAS to probe molecular orientation of semiconducting oligothiophenes such as $\alpha$-sexithiophene [80-82], polythiophenes [20,83-85], and low-bandgap donor-acceptor polymers [20,84,86]. Assigning the transitions near the sulfur K edge is difficult not only because of the limited body of work published, but also due to challenges such as $\pi^{*}$ and $\sigma^{*}$ peaks being very close in energy. For example, thiophene-based molecules exhibit $1 \mathrm{~s} \rightarrow \pi^{*}$ and $1 \mathrm{~s} \rightarrow \sigma^{*}(\mathrm{C}-\mathrm{S})$ transitions that are very close in energy and hard to distinguish experimentally $[80,87,88]$. Complementary XAS simulations have seen only minimal use for the sulfur edge [86,88], but the framework based on DFT used for other elemental edges, like carbon and nitrogen, works well at the sulfur $\mathrm{K}$ edge as well. Additionally, the L edge of sulfur (about $160 \mathrm{eV}-190 \mathrm{eV}$ ) is in an energy range accessible at soft X-ray beamlines and offers further opportunities to understand electronic structure and molecular ordering $[88,89]$. However, L edge calculations pose added challenges and is an area that would benefit from further developments. Predictions of L edge spectra require treatment via full relativistic spin-orbit coupling and accurate description of the relaxation of a core-hole electron, which is difficult for conventional plane-wave DFT methods [90].

Resonant scattering near the sulfur $\mathrm{K}$ edge has potential for providing complementary structural information of polymers and soft matter. It has proven useful in elucidating the internal structure of liquid crystals [91-93]. Resonant scattering at the sulfur K edge has rarely been used in polymers, and only proof of principles has been demonstrated [94]. There is room for growth in this area not only for experimental development, but also to expand our understanding of materials properties that may be revealed through theory and experiment in this regime of relevant X-ray energies.

\subsection{Simulating resonant $X$-ray scattering patterns}

Scattering can be more challenging to interpret because information about characteristic length scales related to molecular packing, orientation, and morphology are also encoded in scattering patterns. Simulations of scattering patterns in the hard Xray regime, where scattering contrast is dominated by differences in electron density, have become prevalent [66,95-97], but very little has been done to predict soft X-ray scattering for polymer and soft material systems. The origins of anisotropic scattering seen with polarized incident X-rays has been explored for a rigidrod type conjugated polymer system [75]. Gann et al. simulated two-dimensional scattering patterns for various morphologies for a blend of a rigid-rod polymer, such as poly(3hexylthiophene) (P3HT), and an isotropic fullerene-like molecule (PCBM). The morphologies included spherical domains of one component in a matrix of the other, and vice versa, as well as a fibrillar polymer structure in an amorphous matrix. The orientation of the polymer chains relative to the fullerene domain at the polymer-fullerene interface was also varied. The simulations alone can reproduce similar anisotropic scattering patterns (Fig. 4) and help understand experimental observations [75]. This demonstrates how scattering patterns can be predicted based on a known morphology, but determining the precise morphology of a sample with unknown structure remains much more challenging.

Scattering experiments performed in transmission geometry typically probe in-plane structure, but variation of incident angle in combination with the contrast enhancement near resonance can allow for accurate determination of depth-dependent morphologies in ordered systems. This has been demonstrated for selfassembled block copolymer thin films, where resonant criticaldimension small angle X-ray scattering combined with thorough data fitting reveal the specific domain morphology of vertical lamella through the film thickness [98,99].

\subsection{In situ and time-resolved studies for dynamics and chemical kinetics}

Advancements in polymer and soft matter based applications and technologies often depend on an understanding of how chemistry and morphology evolve in response to specific processing conditions, for example, thermal annealing, strain, solvent evaporation, or electrical biasing. Although examining structure pre- and post-processing is critical, a full understanding of structure development and chemical changes requires advanced in situ or operando characterization that can probe dynamics and chemical kinetics. In situ and time-resolved investigations are more frequently done with hard X-rays [66,100-103] since experiments do not need to be done under vacuum at these energies. The high vacuum chambers used for soft X-ray experiments makes in situ studies much more challenging, and further complications arise for scattering due to limitations on sample-detector distance.

The growing capability to quickly collect large data sets, especially for in situ experiments, creates a need for fast and even realtime data processing methods. This is actively being developed, especially for high throughput hard X-ray scattering beamlines. Packages such as HipGISAXS connect data collected at beamlines to supercomputers for on-the-fly analysis and modeling [95,104,105], opening new doors to increasing the efficiency of synchrotron experiments and understanding experimental results. Similar advancements are needed to complement soft X-ray techniques as high-throughput, in situ, and more complicated experiments become the norm.

\subsubsection{In situ X-ray absorption spectroscopy}

Simulations of core-level spectroscopy have already proven critical in elucidating changes in chemical bonding in situ. Metalorganic frameworks (MOFs) are coordination polymers with inherent pores from the cage-like structure that can be appended with certain chemistries, such as an amine functionality, to selectively adsorb $\mathrm{CO}_{2}$ to great capacity, and possibly serve as a catalytic center to derive useful chemicals from the greenhouse gas. MOFs 


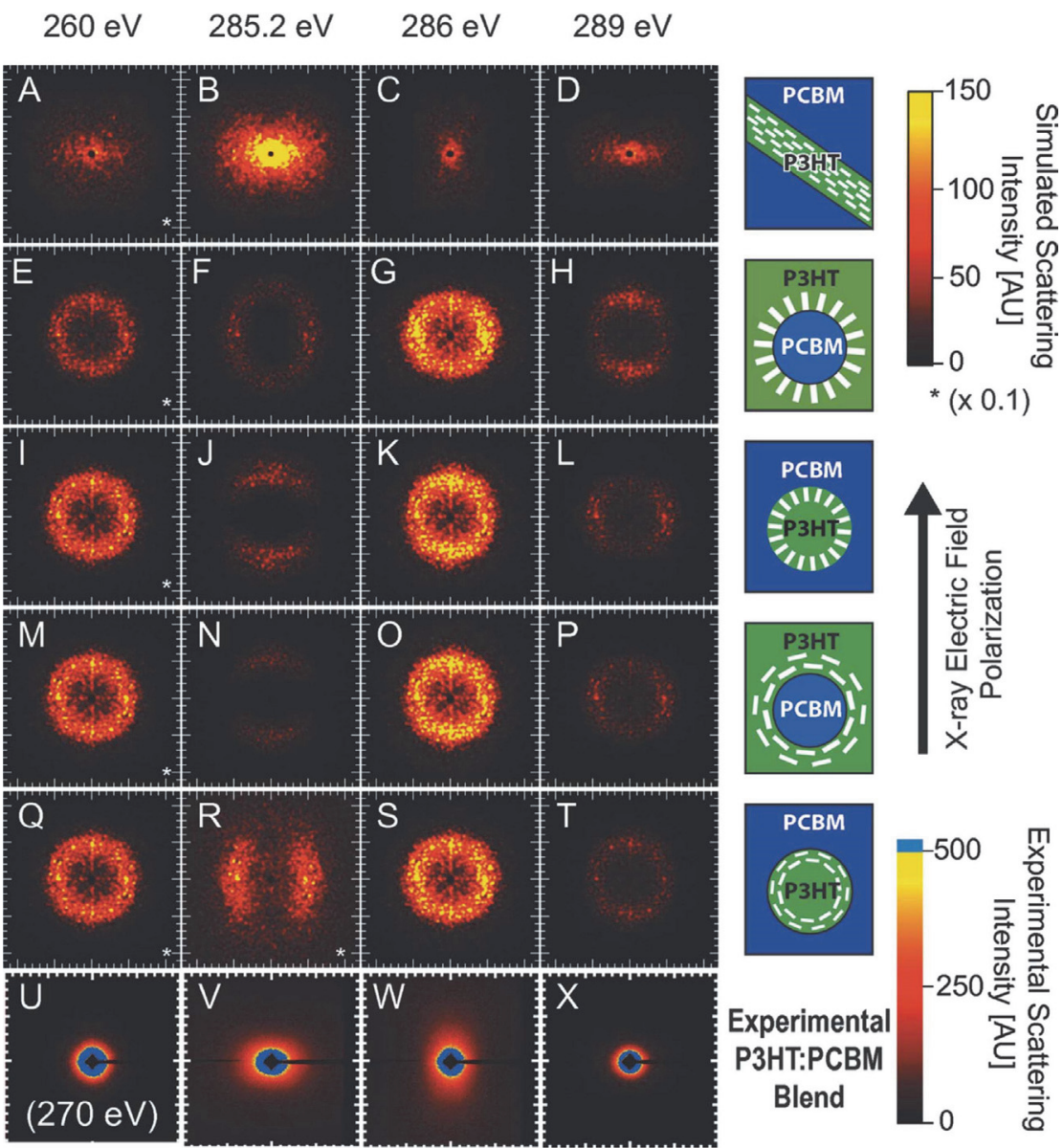

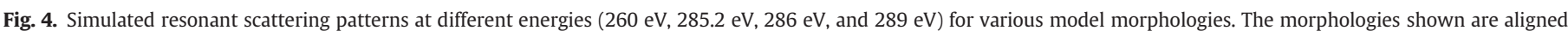

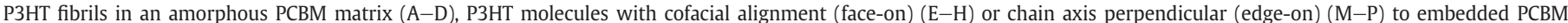

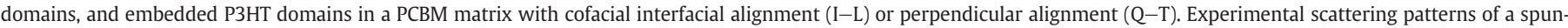

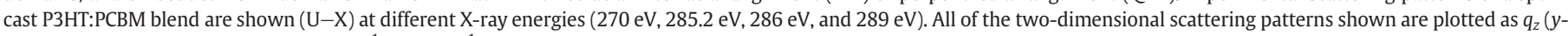
axis) vs. $q_{y}$ ( $x$-axis) ranging from $-1 \mathrm{~nm}^{-1}$ to $1 \mathrm{~nm}^{-1}$. Reprinted from Ref. [75].

have received immense research attention, and recent developments have focused on the in situ monitoring of gas sorption in these hybrid organic membranes from a structural and electronic point of view. Recent work on a diamine-appended MOF reveals a unique 'phase-change' that occurs upon adsorption of $\mathrm{CO}_{2}$. This leads to large $\mathrm{CO}_{2}$ separation capacities that can be attained with small temperature swings and low regeneration energies. This behavior is attributed to the formation of ammonium carbamate chains that form along the pores of the MOF due to adsorbed $\mathrm{CO}_{2}$ molecules that insert into metal-nitrogen bonds [106]. This unique chemistry was determined based on X-ray spectroscopy measurements performed in situ at the nitrogen and oxygen $\mathrm{K}$ edges with varying amounts of $\mathrm{CO}_{2}$ and compared to first-principles predictions on different adsorption structures. Theory verifies the specific bonding configuration needed to produce the observed changes in the NEXAFS spectra. In this case, the carbamate formed has a quasi-trigonal planar nitrogen and is bound to the metal through the oxygen atom [107], as shown in Fig. 5. This underscores the need for predictive calculations to understand the subtle chemical and bonding environment changes that can be revealed by NEXAFS. The tunable energies in RSoXS make it also intrinsically sensitive to chemistry in addition to characteristic length scales and in situ RSoXS studies are needed to reveal information not easily probed by hard X-ray scattering and for continued progress to understand the fundamental processes related to relevant applications.

\subsubsection{Probing changes in interfacial structure and chemistry}

Resonant reflectivity and scattering are useful tools for investigating interfacial phenomenon such as interfacial width in polymers and molecular orientation at domain interfaces as discussed in Sections 2.3 and 3.2. These capabilities lend themselves to future work that understands interfacial processes under operating conditions or as a function of time, building off capabilities of in situ NEXAFS as discussed in Section 3.3.1. For example, in a block copolymer, it is known that a neutral solvent that is equally soluble in both blocks reduces the non-favorable interactions between blocks $A$ and $B$, lowering the Flory-Huggins interaction parameter, $\chi_{A B}$. The interfacial width, $a_{I}$, is related to $\chi_{A B}$ by

$a_{I}=\frac{2 b}{\sqrt{6 \chi_{A B}}}$

where $b$ is the segment length [108-110]. The energy tunability of RSoXS allows chemical sensitivity between distinct blocks, and this can be leveraged to selectively probe interfaces. The value of the invariant, $Q$ defined for an isotropic material as

$$
Q=\frac{1}{2 \pi^{2}} \int_{0}^{\infty} q^{2} I(q) d q
$$

is reduced for diffuse phase boundaries relative to sharp interfaces 

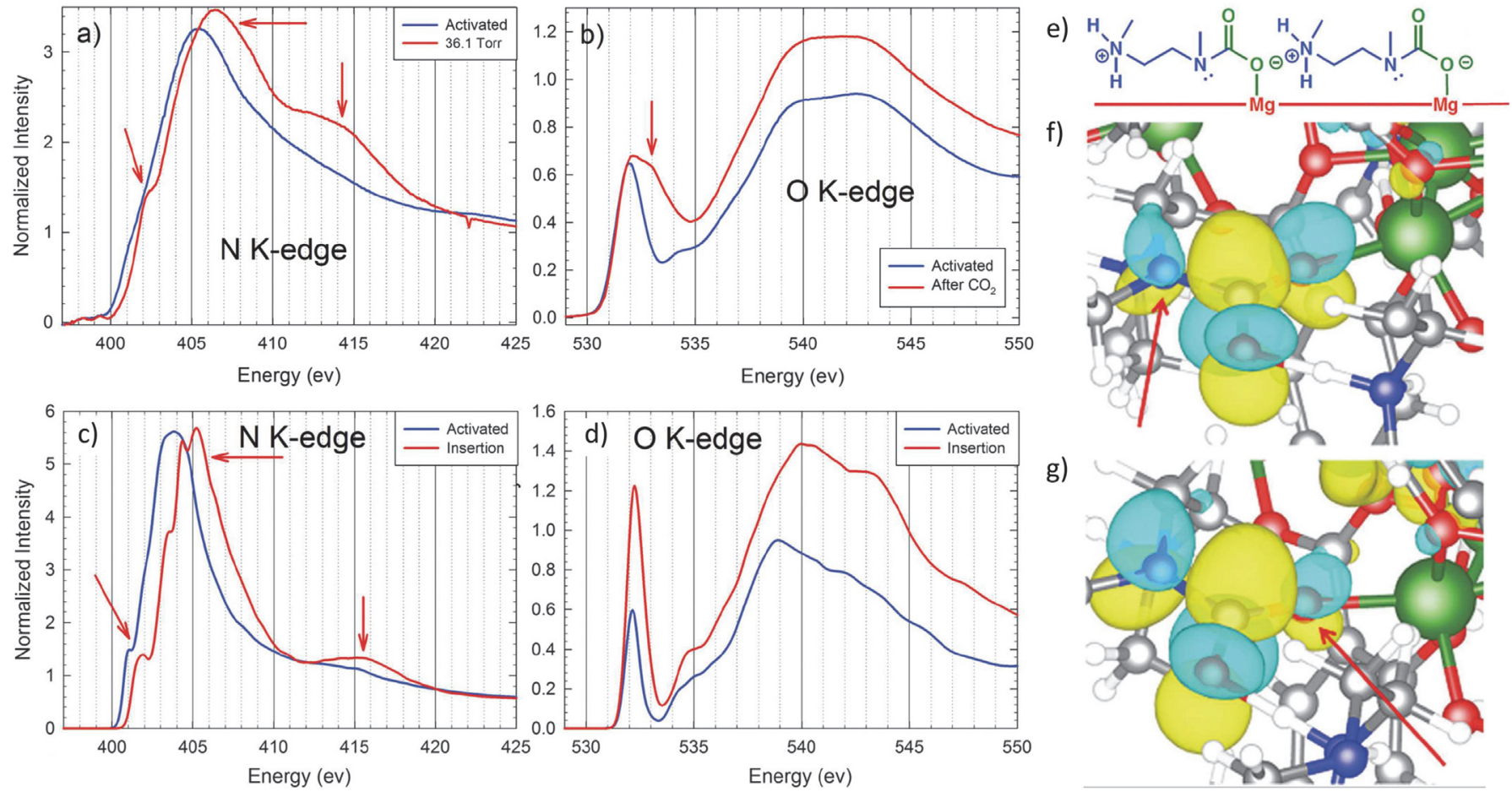

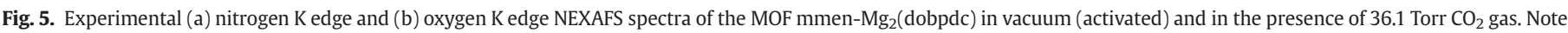

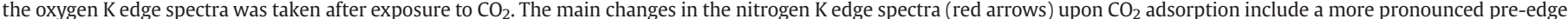

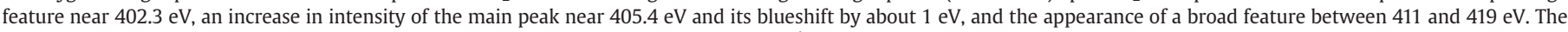

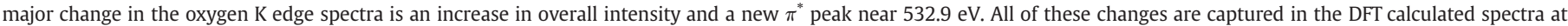

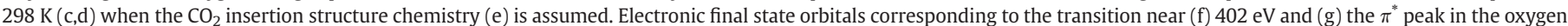

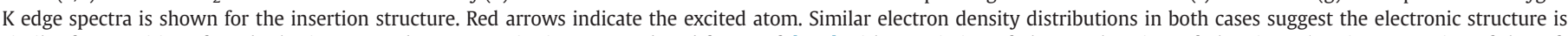

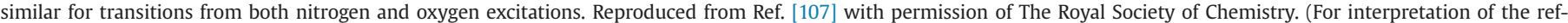
erences to colour in this figure legend, the reader is referred to the web version of this article.)

[111]. Therefore, the invariant can be used to track changes in interfacial width as a function of various processing parameters. Similar to systems studied previously, cylinder forming polystyrene-block-poly(2-vinyl pyridine) (PS-b-P2VP) $(\mathrm{Mn}=40-b$ $18 \mathrm{~kg} / \mathrm{mol}$ ) exposed to tetrahydrofuran (THF) (a nearly neutral solvent) in order to improve long-range order is shown in Fig. 6 [101]. This demonstrates the utility of using XAS and RSoXS to track not only interface localized changes in structure, but also changes in chemistry under in situ conditions.

\subsubsection{Time-resolved dynamics with photon correlation spectroscopy}

The time scales and dynamics of many polymers are dominated by complex rheological processes spanning length scales from nanometers to micrometers. In addition, the hierarchical internal structure inherent to many polymeric materials causes them to flow over long timescales, but respond elastically on much shorter timescales [112]. This behavioral crossover can span several orders of magnitude, thus calling for experimental techniques that are equally adept at accessing dynamic information over many scales. Recently, a coherent scattering technique known as photon correlation spectroscopy (PCS) has been increasingly applied to the X-ray photon regime and is an exceptionally well-suited tool to interrogate polymer dynamics over such scales. In short, PCS provides information on the dynamics of a given system by measuring intensity fluctuations within the scattering patterns that are produced by a coherent source. It is often subdivided into two categories based on the photon energy: dynamic light scattering (DLS), which uses visible light generated by a laser to monitor motion and measure particle sizes down to the sub micrometer- range; and X-ray photon correlation spectroscopy (XPCS), which uses X-rays to probe the dynamics of nanoscale inhomogeneities over a wide range of timescales (about $10^{-3}$ to $10^{4}$ seconds) that are not easily accessible with other techniques (Fig. 7) [113-116].

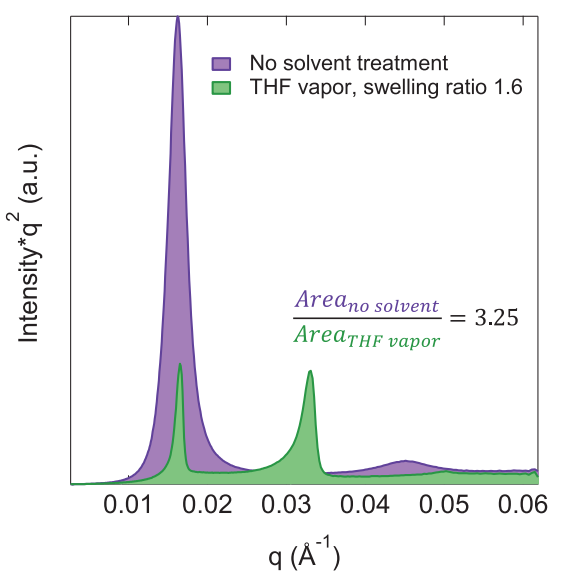

Fig. 6. Circularly averaged transmission resonant X-ray scattering data of a PS- $b-P 2 V P$ block copolymer taken at $285.2 \mathrm{eV}$, where good contrast exists due to the dominant absorption from PS. Data is plotted as $I q^{2}$ vs. $q$ and the area under the curve is related to the invariant, $Q$. The green trace represents a sample that was exposed to THF solvent vapor until a swelling ratio (thickness of swollen film divided by original thickness) of 1.6. The THF vapor leads to a reduction in $\chi_{A B}$ since THF is a nearly neutral solvent for PS and $\mathrm{P} 2 \mathrm{VP}$, and better long-range order is indicated by the sharper, multiple order peaks. However, the reduced $\chi_{A B}$ leads to more diffuse interfaces, revealed through resonant scattering by a reduction of the area under the curve. (For interpretation of the references to colour in this figure legend, the reader is referred to the web version of this article.) 


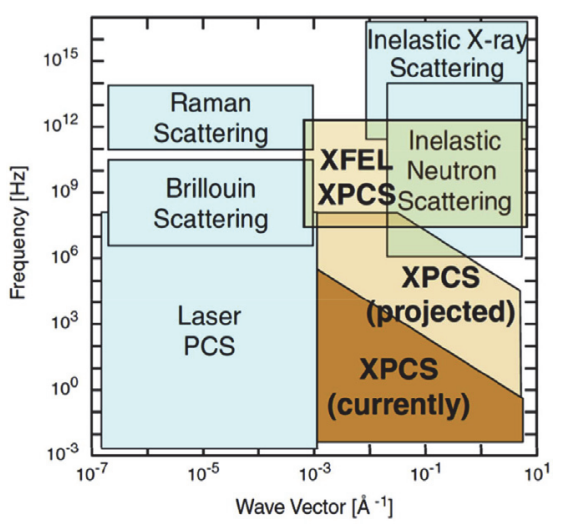

Fig. 7. Plot showing the frequency-scattering wave vector domains accessible by various spectroscopic dynamic techniques. Special emphasis is placed on differentiating the current status of XPCS from the projected improvements upon completion of upgrades to synchrotron facilities. Reprinted from Ref. [112], Copyright 2012, with permission from Elsevier.

However, in order to understand the role that modeling and theory play in realizing the potential of XPCS to study polymer dynamics, it is important to review certain fundamental concepts such as the differences between coherent and incoherent scattering; the essential theory behind the autocorrelation analysis used to dissect the subtle fluctuations in the time-dependent scattering data; and the experimental conditions required to produce a useful scattering plot. First, the scattering patterns from incoherent X-rays represents a statistical average over many incoherent electron density regions within the sample, which gives rise to an isotropically-broadened scattering peak. On the other hand, coherent X-ray experiments produce a scattering pattern with peaks composed of specific subregions with increased intensity, or speckles. These speckles are formed as a result of the mutual constructive interference of two X-ray wavefronts, which originate from a given scattering point. Thus, their inverse Fourier transform can give an electron density distribution with nanometer precision.

Snapshots of speckle patterns are taken at various time intervals, where their sequence reveals subtle intensity, $I$, fluctuations that can be analyzed by a second order autocorrelation function, $g_{2}$, to extract correlation times, $\tau$, characteristic to each wave vector, $Q$ according to the following relation,

$$
g_{2}(t)=\frac{\langle I(\tau) I(\tau+t)\rangle}{\langle I(\tau)\rangle^{2}}=1+A|F(Q, t)|^{2}
$$

where the time spacing of the speckle sequence, $\Delta t$, determines the timescale of the dynamics probed. Finally, assuming a system with stationary dynamics around an equilibrium (e.g. Brownian motion), a plot can be obtained as shown in Fig. 8 where the intermediate a

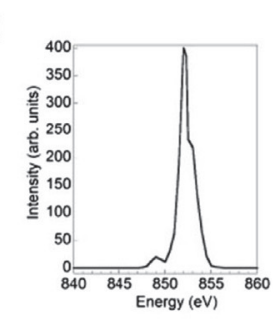

b

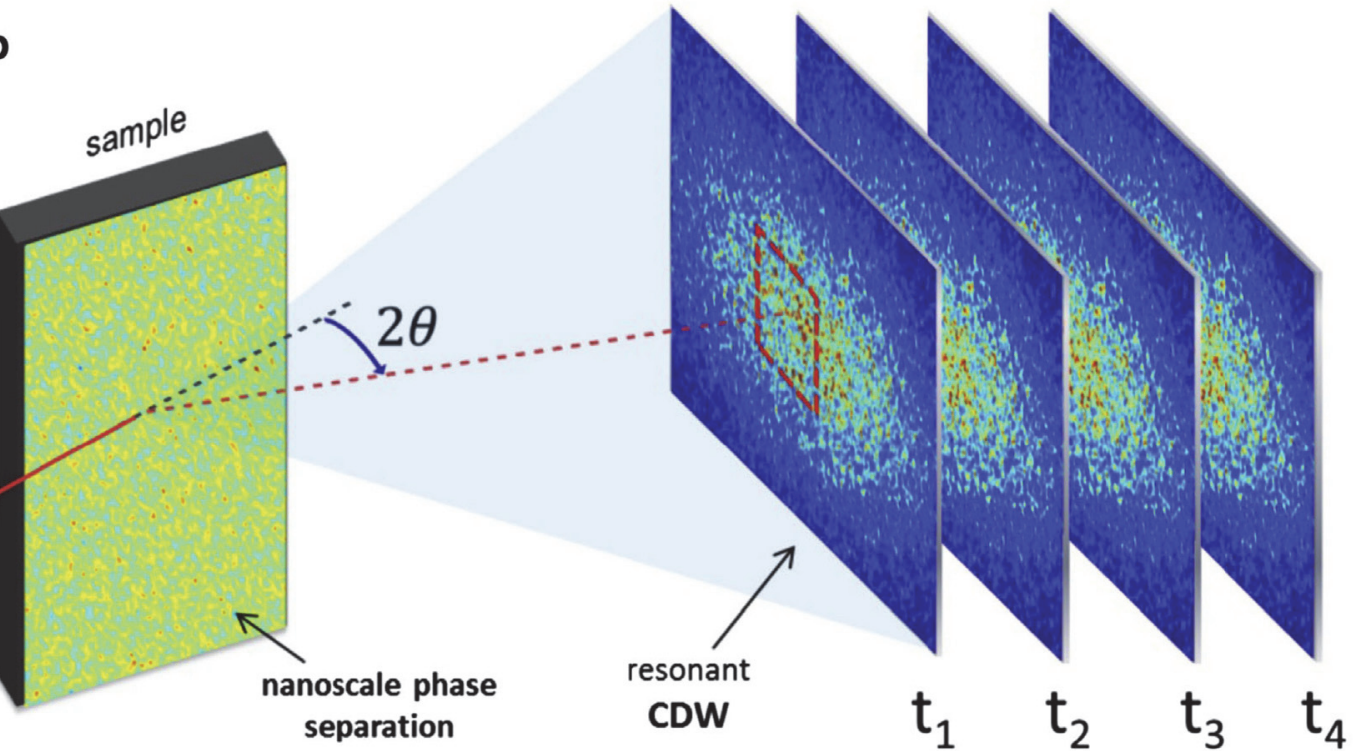

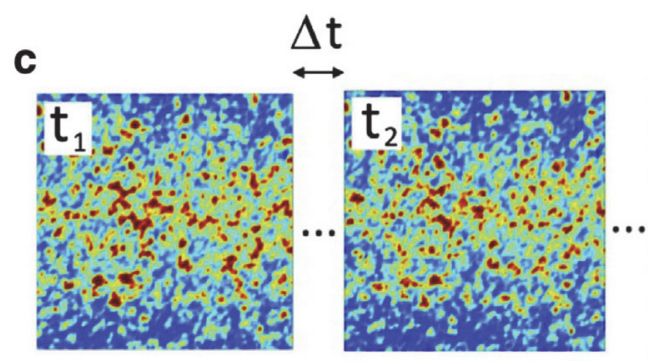
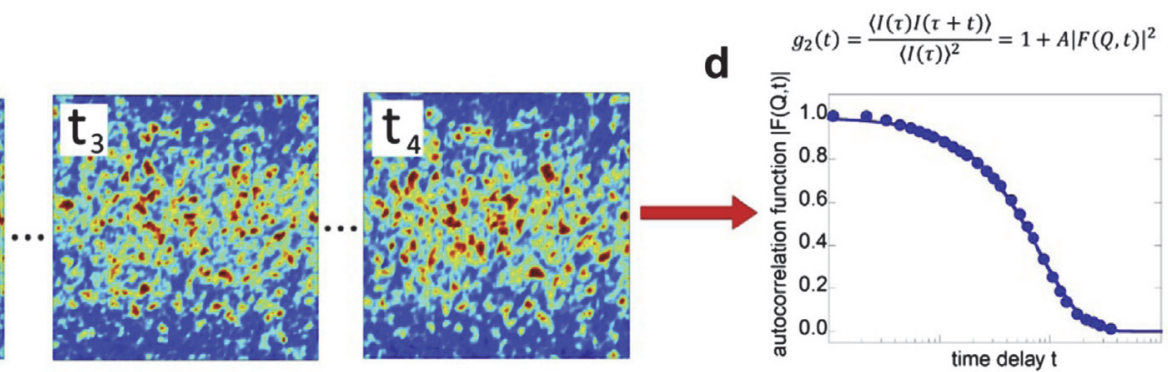

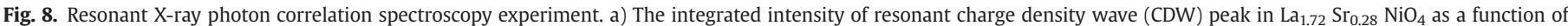

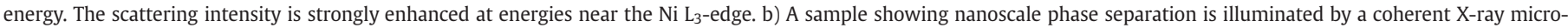

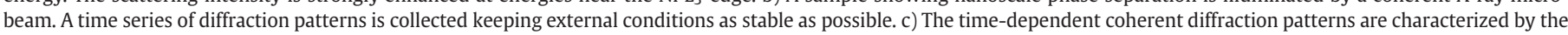

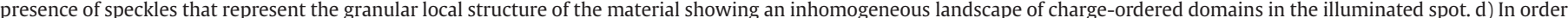

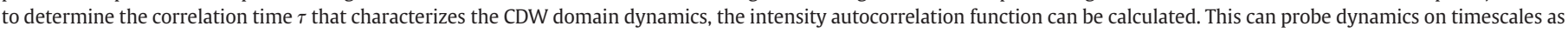
short as the time spacing $\Delta t$ of the collected time series. Reproduced with permission from Springer [136]. 
scattering function, $A|F(Q, t)|^{2}$, can be modeled to extract dynamic parameters.

Experimentally, the well-established autocorrelation theory described above can only be applied if these scattering events occur only once (limiting the thickness/density of the sample) and if the ergodic principle, which assumes that no information is lost within each snapshot time, is satisfied (limiting the short timescale resolution). Multiple scattering can be avoided by using a small micrometer sized sample (such as a single crystal). On the one hand, this can be very useful because it circumvents the requirement of a small slit to induce coherence since the sample itself will be the source. On the other hand, this approach severely limits the range of samples that can be used.

This divergence between the samples and length scales that are accessible are what distinguish the applicability of XPCS from DLS. Currently, DLS is used on a routine basis for the analysis of particle sizes in the sub micrometer range; it provides an estimation of the average size and its distribution within a measuring time of a few minutes. However, improvements in the ability to model dynamic behavior and relate it to scattering data has continued to make it a useful tool to study slow effects such as swelling mechanisms and phase transitions [117-127]. Still, XPCS is considered superior to DLS in certain situations because 1 ) it enables high- $q$ dynamics and 2 ) it can probe samples that are opaque or subject to multiple light scattering phenomena under DLS conditions. For example, the high $q$-range of XPCS can be used to extract elusive surface dynamics such as polymer chains and the relaxation rates of capillary waves on surfaces by directly probing a material in a grazing incidence geometry [112,128-132].

There are also many samples exhibiting long-term relaxations of interest, like "jamming systems," where the only way to distinguish slow sample movements from random fluctuations is by analyzing the slow drift of the speckle pattern. Such slow drifts cannot be observed in a DLS experiment due to the beam size, while the usual $\mu \mathrm{m}$-scale beam used in XPCS can probe such flows. Therefore, several methods developed for light scattering experiments are now used with X-rays. One example is a method called heterodyning that can be used to obtain interferences between a reference and the sample. This provides correlation functions where heterodyning a sample's diffracted amplitude against that of a reference can be used for phase retrieval, separate fluctuations from long-term flows [133], and mechanical relaxation from aggregate diffusion [134].

Another challenge is studying polymer dynamics in the $\mu \mathrm{s}$ to $\mathrm{ms}$ regime, which will require sources with greater coherent flux in order to obtain a useful signal to noise ratio. The recent push to improve coherence of many synchrotrons is an exciting development in this regard [135]. Access to this temporal regime will enable a deeper understanding of polymer systems away from equilibrium or near the onset of more complex dynamical heterogeneities. The detailed quantification of such phenomena is critical to elucidating the arrested states of systems. However, the autocorrelation analysis of the data produced by such transient states will require use of a more involved two-time normalized correlation function $C\left(q, t_{1}, t_{2}\right)$ with normalized variance and fourth-order (as opposed to traditional second order) autocorrelation functions. These complex autocorrelation quantities will result from transient speckle scattering patterns whose origin is likely to be chemical in nature. Therefore, the development of XPCS capabilities in this temporal regime will also rely heavily on complementary theoretical modeling of the absorptive and scattering properties of the polymer materials under investigation.

Future work must explore the dynamics of specific chemistries. This will help achieve a better understanding of structure-property relationships and selectively probe the dynamics of buried interfaces. Once again, this can be achieved by combining XPCS with spectroscopy. However, most of the resonance-enhanced XPCS research up this point has been done using soft X-rays on solid inorganic materials (Fig 8) [136-141]. Still, despite the thorough background shown in the previous sections, resonanceenhanced XPCS has not seen much use in polymer science.

Since scientific content in XPCS often derives from the deviation of the intensity correlation function from simple exponential line shapes, reliable and precise line shape theoretical analysis is fundamental to facilitating the use of theory to rationalize XPCS results. As synchrotron sources improve their coherent flux, the improved signal to noise ratios will enable researchers to reach even shorter timescales and probe more weakly scattering materials. Athough this will enable unique insights into a new realm of material dynamics and in-situ/operando experiments, understanding of such studies will hinge even more upon the presence of a solid foundation linking scattering and spectroscopy theory with experiments.

\section{Applications and outlook}

The co-development of theoretical and experimental tools for soft X-ray absorption spectroscopy and scattering is focused on characterizing soft materials undergoing dynamic changes in relevant device and material application environments. In this section, two key areas of state-of-the-art polymer science that can greatly benefit from this combined theoretical and experimental approach of in situ soft X-ray studies are highlighted: polymer materials for gas storage, ion conduction, water filtration membranes, and doped conjugated polymers for use in organic electronics.

\subsection{Polymer-based membranes}

For membrane materials in applications of fuel cells, batteries, $\mathrm{CO}_{2}$ capture with hybrid organic frameworks, and water filtration, it is critical to not only analyze the morphology of the material designed and fabricated to assess its suitability for diffusion of ions, gas, and water through its pores, but also to develop understanding for how the material behaves structurally in situ. The use of soft Xray spectroscopy and scattering enable the contrast-enhanced probing of electronic structure and molecular to mesoscale material structure in complex blends of polymers and organic small molecules. The low energy of soft X-rays is critical to generating enhanced contrast between similarly low- $Z$, carbonaceous components that only differ slightly in local chemical bonding environment. In the simplest case of polymers of intrinsic microporosity (PIMs), the membrane is composed of a neat polymer, and the contrast is derived between the polymer and vacuum (which comprises the pores when under vacuum conditions for measurement). These porous polymers and their very small $(<2 \mathrm{~nm})$ intrinsic pores are ideal structures for gas storage and separation [5,7]. Essentially, the neat polymer comprises the matrix and provides mechanical integrity to the membrane, while the 'unoccupied' volume that arises from imperfect packing of rigid polymer backbones serves as the 'highways' for transport of ions, gas, or water, depending on the chemistry of the polymer. One time-dependent study using small angle (SAXS) and wide angle Xray scattering (WAXS) and molecular dynamics (MD) simulations probes the dynamics of aging in microporous polymers with pore size $<1 \mathrm{~nm}$ [142]. Physical aging is relevant because the closing of pores via this mechanism can drastically reduce molecular transport. Runt et al. found that there are two distinct aging mechanisms in PIMs: decrease of pore volume fraction followed by pore shrinkage. In another strategy for creating pores in polymer films 
for mesoporous conducting and storage membranes, inherently templated polyethylene- $b$-polystyrene- $b$-polyethylene (SES) films are impregnated with hPS and rinsed with a selective solvent to generate pores within the PS domains that can be further treated by sulfonation to enhance hydrophilicity and ion conduction $[74,143,144]$. The structural analysis that is key to ion conduction will probe the location, size, and fraction of each block component relative to the generated voids, and RSoXS has been shown by Balsara and coworkers to be the ideal tool for characterizing these pores in heterogeneous soft matter membranes. Without the contrast-matching resonance of RSoXS, it would not be possible to distinguish between blocks and determine the relative locations of the pores and hydrophobic and hydrophilic domains. Similarly, the same group has mixed $\mathrm{TiO}_{2}$ nanoparticles into templated thin films of PS- $b$-PEO battery membranes in an attempt to block dendrite runaway growth during electrochemical cycling [145]. Using RSoXS and mechanical testing, it was found that there exists an ideal nanoparticle loading near $20 \mathrm{wt} \%$ that preserves the block copolymer ordering and optimizes the membrane toughness. This type of study could be extended to operando RSoXS experiments where membranes are cycled electrochemically, and the changing morphology near the block copolymer domains and nanoparticle interfaces can be probed.

Nafion is a well-studied material with a complex microstructure that is suitable for fuel cell, water filtration, and solar-fuels generation membranes. It forms a heterogeneous structure in which parallel, randomly packed, inverted micellar cylinders are comprised of a polytetrafluoroethylene (PTFE) backbone on the outside and hydrophilic ionic side chains on the inside, forming a hydrophilic cylindrical micelle core that enables water and ions to be transported efficiently [146]. In situ grazing incidence SAXS (GISAXS) has been used to study the existence of cylindrical domains of Nafion and how they swell during water treatment. Segalman et al. point out the importance of the substrate interface on directing either in-plane only or random orientation of cylinders; and it is found that a random mixture is better to maximize water, oxygen, and ion transport. Thermal annealing during GISAXS revealed that water uptake is restricted following extensive thermal processing due to the formation of Nafion crystals that begin to take away from the compressibility of the Nafion matrix that allows substantial water uptake. The authors are able to monitor the swelling of the Nafion pores with water in real time with GISAXS [147]. Similarly, in situ SAXS on Nafion membranes in the bulk shows an enormous difference in time scales of water uptake and swelling for membranes treated with liquid versus water vapor. From these studies, the $d$-spacing of the 'ionomer' peak is observed, which represents the spacing between randomly packed inverse micellar cylinders of water transporting Nafion [148]. Some new preliminary RSoXS results reveal that the polymer chains are partially oriented inside ionomer domains (inverse micelles) near the interface with adjacent crystallites, and that this orientational ordering occurs even before the crystalline phase begins to form [65]. Future studies should continue to use RSoXS to probe the orientational and ionomer domain ordering, but in a time-resolved in situ study in aqueous environments with various salt and $\mathrm{pH}$ treatments. Using a solution environment cell that is currently under development, such studies will help to determine the molecular details of ionomer domain formation and to visualize the swelling of these membranes during operation.

\subsection{Doped semiconducting polymers}

The doping of conjugated polymers is a topic that has attracted much interest, but unanswered questions remain regarding the relationship of dopant-polymer microstructure (dopant incorporation), ionization efficiency and resulting device enhancement. The combination of X-ray absorption spectroscopy and resonant scattering have potential to probe changes in electronic structure and corresponding spatial distributions that occur upon doping and help understand charge transfer.

The use of first-principles calculations is especially needed to understand details of electronic structure in conjugated polymers and small molecules. Organic semiconductors can have distinct NEXAFS spectra due to unique electronic structure characteristics. For example, a biradical hydrocarbon that exists as a stable radical with an unpaired electron in a singly occupied molecular orbital reveals a very low energy NEXAFS peak (283.4 eV), which is attributed to its low-lying lowest unoccupied molecular orbital [149]. Comparing experimental spectra to simulations can help determine where on the polymer backbone (or small molecule) an excited state exists, and how the dopant may change the local chemical bonding environment [51]. There have been many examples of doping in conjugated polymers [150], using varied dopants from organic small molecules to graphene oxide [151] and ranging in application from organic thin film transistors to sensors to devices for the tissue-electrode interface in biology.

Efforts seek to understand how the dopant-host structure determines ionization processes and efficiency. For example, the electronic structure of polyaniline (PANi) was investigated by XAS at various doping levels by organic acids. It was found with this soft X-ray strategy that doping occurs by protonation of the backbone nitrogen sites of PANi and changes the local chemical bonding configuration by breakup of the quinoid structure and disappearance of imine bonding, leaving only amine bonding characteristics [152]. $\mathrm{Cu}$ and Fe K edge NEXAFS were utilized in a different doping strategy to study the in situ electrochemical inclusion of $\mathrm{Cu}$ and $\mathrm{Fe}$ species in poly(3-methylthiophene) (P3MT). It was found that the $\mathrm{Cu}$ ion is an effective dopant for P3MT through its complexation with the sulfur atom within the backbone, following the reversible, time-dependent $\mathrm{Cu}^{I I}-\mathrm{Cu}^{I}-\mathrm{Cu}^{0}$ reduction process [153]. Analogously, NEXAFS was combined with DFT to probe local conformation and electronic structure of P3HT doped with F4TCNQ a common p-type dopant for organic semiconductors. It was found that the dopant is inserted into some crystallites of P3HT in a planar fashion such that cofacial stacking of the two species occurs [154]. Another study confirmed that only some crystallites contain the dopant, and that weak and strong doping regimes occur [150]. In a similar polymer system, PBTTT- $\mathrm{C}_{14}$, in which the thiophene monomers of P3HT are spaced farther apart by a thienothiophene unit, in situ GIWAXS monitored the incorporation of the F4TCNQ dopant [155]. A similar cofacial arrangement was determined that preserves planarity of the polymer backbone within crystallites, but this time the polymer crystallites all contain the dopant, and in nonrandom, correlated locations. This correlated, nonrandom arrangement of dopants throughout the semiconductor crystallites and film has strong implications for transport models due to the nature of charge hopping in these 1D-like semiconductors. The dopants in this study were also found to cause crystallite contraction in the $\pi$-stacking direction ( $b$ axis) and slight expansion and paracrystalline disorder in the alkyl-stacking direction ( $a$ axis). This well-thought study should be extended to other polymers and dopants, for example using in situ ion gating where doping reversibility and effect of electrochemistry can be investigated.

Challenges exist since a theoretical basis to predict changes in Xray absorption spectra for molecules that have donated or accepted an electron is still not well developed. This is needed to move forward in understanding the spatial distribution not only of the dopant [150], but also of electrically doped sites within an organic 
semiconductor, which could be probed through resonant scattering, even during device operating conditions.

\section{Conclusions}

X-ray absorption spectroscopy and resonant scattering and reflectivity are useful tools for combined chemical and spatial analysis of polymers and soft materials. Along with the benefits that these techniques provide, challenges also exist that must be overcome; including an improved understanding at the atomic level of electronic transitions, polarization and orientation dependent absorption and scattering properties, and an ability to predict resonant scattering patterns of intricate systems. As more complex material systems and advanced in situ and operando experiments become essential to improve understanding related to various applications, addressing these challenges becomes more necessary to take full advantage of the capabilities of X-ray spectroscopy and resonant scattering. In the future, complementary calculations will be more closely integrated to experiments to predict and analyze data and accelerate materials development.

With this combination of theory and soft X-ray spectroscopy and scattering, soft X-rays will prove to be an essential part of the analytical toolkit for polymer scientists, especially with the ability to conduct time-resolved, in situ XPCS experiments that track distinct scattering centers under relevant sample conditions. Two key application areas of soft matter, doping of conjugated polymers and polymer membrane structure, were discussed both in terms of the morphological and electronic structure understanding developed thus far, and the potential power that soft X-ray spectroscopy and scattering have in determining a more complete picture for these materials. Many other systems exist where the combination of soft X-rays and electronic structure modeling will be essential in studying the details of dynamic structure of soft matter under operating conditions.

\section{Acknowledgments}

This work was supported by the Advanced Light Source (ALS) and the Molecular Foundry, user facilities located at the Lawrence Berkeley National Laboratory and supported by the Director, Office of Science, Office of Basic Energy Sciences, of the U.S. Department of Energy under Contract No. DE-AC02-05CH11231. The authors thank Xiaodan $\mathrm{Gu}$ for helpful discussions.

\section{References}

[1] H. Sirringhaus, 25th anniversary article: organic field-effect transistors: the path beyond amorphous silicon, Adv. Mater. 26 (2014) 1319-1335, http:/ dx.doi.org/10.1002/adma.201304346.

[2] L. Dou, J. You, Z. Hong, Z. Xu, G. Li, R.A. Street, et al., 25th anniversary article: a decade of organic/polymeric photovoltaic research, Adv. Mater. 25 (46) (2013) 6642-6671, http://dx.doi.org/10.1002/adma.201302563.

[3] S. Holliday, J.E. Donaghey, I. McCulloch, Advances in charge carrier mobilities of semiconducting polymers used in organic transistors, Chem. Mater. 26 (1) (2014) 647-663, http://dx.doi.org/10.1021/cm402421p.

[4] Y. Huang, E.J. Kramer, A.J. Heeger, G.C. Bazan, Bulk heterojunction solar cells: morphology and performance relationships, Chem. Rev. 114 (14) (2014) 7006-7043, http://dx.doi.org/10.1021/cr400353v.

[5] C. Xu, N. Hedin, Microporous adsorbents for $\{\mathrm{CO} 2\}$ capture - a case for microporous polymers? Mater. Today 17 (8) (2014) 397-403, http:/ dx.doi.org/10.1016/j.mattod.2014.05.007.

[6] B. Sreenivasulu, I. Sreedhar, P. Suresh, K.V. Raghavan, Development trends in porous adsorbents for carbon capture, Environ. Sci. Technol. 49 (21) (2015) $12641-12661$.

[7] N. Du, H.B. Park, G.P. Robertson, M.M. Dal-Cin, T. Visser, L. Scoles, et al., Polymer nanosieve membranes for $\mathrm{CO}_{2}$-capture applications, Nat. Mater. 10 (2011) 372-375, http://dx.doi.org/10.1038/nmat2989.

[8] H. Zhang, P.K. Shen, Recent development of polymer electrolyte membranes for fuel cells, Chem. Rev. 112 (5) (2012) 2780-2832, http://dx.doi.org/ $10.1021 / \mathrm{cr} 200035 \mathrm{~s}$.
[9] D.T. Hallinan Jr., N.P. Balsara, Polymer electrolytes, Annu. Rev. Mater. Res. 43 (1) (2013) 503-525, http://dx.doi.org/10.1146/annurev-matsci-071312121705.

[10] W.S. Young, W.F. Kuan, T.H. Epps, Block copolymer electrolytes for rechargeable lithium batteries, J. Polym. Sci. B Polym. Phys. 52 (1) (2014) 1-16, http://dx.doi.org/10.1002/polb.23404.

[11] Z. Nie, E. Kumacheva, Patterning surface with functional polymers, Nat. Mater. 7 (2008) 277-290, http://dx.doi.org/10.1038/nmat2109.

12] M.P. Stoykovich, M. Müller, S.O. Kim, H.H. Solak, E.W. Edwards, J.J. de Pablo, et al., Directed assembly of block copolymer blends into nonregular deviceoriented structures, Science 308 (5727) (2005) 1442-1446, http:// dx.doi.org/10.1126/science.1111041.

[13] R. Ruiz, H. Kang F.A. Detcheverry, E. Dobisz, D.S. Kercher, T.R. Albrecht, et al. Density multiplication and improved lithography by directed block copolymer assembly, Science 321 (5891) (2008) 936-939.

14] B. Henke, E. Gullikson, J. Davis, X-ray interactions: photoabsorption, scattering, transmission, and reflection at $\mathrm{E}=50-30,000 \mathrm{eV}, \mathrm{Z}=1-92, \mathrm{~A}$. T. Data Nucl. Data Tables 54 (2) (1993) 181-342, http://dx.doi.org/10.1006/ adnd.1993.1013.

[15] D.A. Outka, J. Stöhr, J.P. Rabe, J.D. Swalen, The orientation of langmuirblodgett monolayers using nexafs, J. Chem. Phys. 88 (6) (1988) 4076-4087, http://dx.doi.org/10.1063/1.453862.

[16] S. Krishnan, R. Ayothi, A. Hexemer, J.A. Finlay, K.E. Sohn, R. Perry, et al., Antibiofouling properties of comblike block copolymers with amphiphilic side chains, Langmuir 22 (11) (2006) 5075-5086, http://dx.doi.org/10.1021/ la0529781.

[17] M.D. Dimitriou, Z. Zhou, H.S. Yoo, K.L. Killops, J.A. Finlay, G. Cone, et al., A general approach to controlling the surface composition of poly(ethylene oxide)-based block copolymers for antifouling coatings, Langmuir 27 (22) (2011) 13762-13772, http://dx.doi.org/10.1021/la202509m.

[18] W. van Zoelen, R.N. Zuckermann, R.A. Segalman, Tunable surface properties from sequence-specific polypeptoid polystyrene block copolymer thin films, Macromolecules 45 (17) (2012) 7072-7082, http://dx.doi.org/10.1021/ ma3009806.

[19] D.M. DeLongchamp, R.J. Kline, D.A. Fischer, L.J. Richter, M.F. Toney, Molecular characterization of organic electronic films, Adv. Mater. 23 (3) (2011) 319-337, http://dx.doi.org/10.1002/adma.201001760.

[20] U. Aygül, H. Peisert, D. Batchelor, U. Dettinger, M. Ivanovic, A. Tournebize, et al., Molecular orientation in polymer/fullerene blend films and the influence of annealing, Sol. Energy Mater. Sol. Cells 128 (2014) 119-125, http:// dx.doi.org/10.1016/j.solmat.2014.05.017.

[21] C.R. McNeill, H. Ade, Soft x-ray characterisation of organic semiconductor films, J. Mater. Chem. C 1 (2013) 187-201, http://dx.doi.org/10.1039/ C2TC00001F.

[22] T. Schuettfort, L. Thomsen, C.R. McNeill, Observation of a distinct surface molecular orientation in films of a high mobility conjugated polymer, J. Am. Chem. Soc. 135 (3) (2013) 1092-1101, http://dx.doi.org/10.1021/ja310240q.

[23] E. Gann, C.R. McNeill, M. Szumilo, H. Sirringhaus, M. Sommer, S. Maniam, et al., Near-edge X-ray absorption fine-structure spectroscopy of naphthalene diimide-thiophene co-polymers, J. Chem. Phys. 140 (16) (2014) 164710, http://dx.doi.org/10.1063/1.4871463.

[24] C.R. McNeill, B. Watts, L. Thomsen, W.J. Belcher, N.C. Greenhamand, P.C. Dastoor, Nanoscale quantitative chemical mapping of conjugated polymer blends, Nano Lett. 6 (6) (2006) 1202-1206, http://dx.doi.org/10.1021/ nl060583w.

[25] C.R. McNeill, K. Asadi, B. Watts, P.W.M. Blom, D.M. de Leeuw, Structure of phase-separated ferroelectric/semiconducting polymer blends for organic non-volatile memories, Small 6 (4) (2010) 508-512, http://dx.doi.org/ 10.1002/smll.200901719.

[26] B. Watts, H. Ade, NEXAFS imaging of synthetic organic materials, Mater. Today 15 (4) (2012) 148-157, http://dx.doi.org/10.1016/S1369-7021(12) 70068-8.

[27] H. Ade, X. Zhang, S. Cameron, C. Costello, J. Kirz, S. Williams, Chemical contrast in X-ray microscopy and spatially resolved XANES spectroscopy of organic specimens, Science 258 (1992) 972-975, http://dx.doi.org/10.1126/ science. 1439809.

[28] H. Ade, A. Smith, S. Cameron, R. Cieslinski, G. Mitchell, B. Hsiao, et al., X-ray microscopy in polymer science: prospects of a new imaging technique, Polymer 36 (9) (1995) 1843-1848, http://dx.doi.org/10.1016/0032-3861(95) 90930-Z.

[29] H. Ade, B. Hsiao, X-ray linear dichroism microscopy, Science 262 (1993) 1427-1429, http://dx.doi.org/10.1126/science.262.5138.1427.

[30] B. Watts, C.R. McNeill, Simultaneous surface and bulk imaging of polymer blends with x-ray spectromicroscopy, Macromol. Rapid Commun. 31 (19) (2010) 1706-1712, http://dx.doi.org/10.1002/marc.201000269.

[31] H. Ade, H. Stoll, Near-edge X-ray absorption fine-structure microscopy of organic and magnetic materials, Nat. Mater. 8 (2009) 281-290, http:// dx.doi.org/10.1038/nmat2399.

[32] P. Hohenberg, W. Kohn, Inhomogeneous electron gas, Phys. Rev. 136 (1964) B864, http://dx.doi.org/10.1103/PhysRev.136.B864.

[33] W. Kohn, L.J. Sham, Self-consistent equations including exchange and correlation effects, Phys. Rev. 140 (1965) A1133, http://dx.doi.org/10.1103/ PhysRev.140.A1133.

[34] P. Elliott, F. Furche, K. Burke, Excited States from Time-dependent Density Functional Theory, chap. 3, John Wiley \& Sons, Inc., 2009, ISBN 
9780470399545, $\quad$ pp. $\quad 91-165, \quad$ http://dx.doi.org/10.1002/ 9780470399545 ch3

[35] D. Prendergast, G. Galli, X-ray absorption spectra of water from first principles calculations, Phys. Rev. Lett. 96 (2006) 215502, http://dx.doi.org/ 10.1103/PhysRevLett.96.215502.

[36] K. Hermann, L.G.M. Pettersson, M.E. Casida, C. Daul, A. Goursot, A. Koester, et al., Stobe-demon Version 3.3, 2014.

[37] M. Magnuson, L. Yang, J.H. Guo, C. Såthe, A. Agui, J. Nordgren, et al., The electronic structure of poly(pyridine-2,5-diyl) investigated by soft X-ray absorption and emission spectroscopies, Chem. Phys. 237 (3) (1998) 295-304, http://dx.doi.org/10.1016/S0301-0104(98)00262-6.

[38] S.G. Urquhart, A.P. Hitchcock, A.P. Smith, H.W. Ade, W. Lidy, E.G. Rightor, et al., NEXAFS spectromicroscopy of polymers: overview and quantitative analysis of polyurethane polymers, J. Electron Spectrosc. Relat. Phenom. 100 (1999) 119-135, http://dx.doi.org/10.1016/S0368-2048(99)00043-2.

[39] L.J. Gamble, B. Ravel, D.A. Fischer, D.G. Castner, Surface structure and orientation of PTFE films determined by experimental and FEFF8-calculated NEXAFS spectra, Langmuir 18 (6) (2002) 2183-2189, http://dx.doi.org/ 10.1021/la0112581.

[40] H. Ågren, V. Carravetta, O. Vahtras, L.G.M. Pettersson, Orientational probing of polymeric thin films by nexafs: calculations on polytetrafluoroethylene, Phys. Rev. B 51 (1995) 17848-17855, http://dx.doi.org/10.1103/ PhysRevB.51.17848.

[41] W. Eberhardt, T.K. Sham, R. Carr, S. Krummacher, M. Strongin, S.L. Weng, et al., Site-specific fragmentation of small molecules following soft-x-ray excitation, Phys. Rev. Lett. 50 (1983) 1038-1041, http://dx.doi.org/ 10.1103/PhysRevLett.50.1038

[42] O. Takahashi, K. Tabayashi, S.i. Wada, R. Sumii, K. Tanaka, M. Odelius, et al., Theoretical study of ion desorption from poly-(methyl methacrylate) and poly-(isopropenyl acetate) thin films through core excitation, J. Chem. Phys. 124 (12) (2006) 124901, http://dx.doi.org/10.1063/1.2176605.

[43] S.N. Patel, G.M. Su, C. Luo, M. Wang, L.A. Perez, D.A. Fischer, et al., NEXAFS spectroscopy reveals the molecular orientation in blade-coated pyridal[2,1,3] thiadiazole-containing conjugated polymer thin films, Macromolecules 48 (18) (2015) 6606-6616, http://dx.doi.org/10.1021/acs.macromol.5b01647.

[44] M.M. Nahid, E. Gann, L. Thomsen, C.R. McNeill, NEXAFS spectroscopy of conjugated polymers, Eur. Polym. J. (2016), http://dx.doi.org/10.1016/ j.eurpolymj.2016.01.017.

[45] J.L. Brédas, D. Beljonne, V.C.J. Cornil, Charge-transfer and energy-transfer processes in $\pi$-conjugated oligomers and polymers: a molecular picture Chem. Rev. 104 (11) (2004) 4971-5004, http://dx.doi.org/10.1021/ Cr040084k.

[46] J. Stöhr, NEXAFS Spectroscopy. Springer Series in Surface Sciences, Springer, 1992, ISBN 9783540544227

[47] D.A. Outka, J. Stöhr, J. Rabe, J.D. Swalen, H.H. Rotermund, Orientation of arachidate chains in langmuir-blodgett monolayers on si(111), Phys. Rev. Lett. 59 (1987) 1321-1324, http://dx.doi.org/10.1103/PhysRevLett.59.1321.

[48] L.G.M. Pettersson, H. Ågren, B.L. Schürmann, A. Lippitz, W.E.S. Unger, Assembly and decomposition of building blocks to analyze polymer nexafs spectra, Int. J. Quantum Chem. 63 (3) (1997) 749-765.

[49] V. Carravetta, H. Ågren, L.G.M. Pettersson, O. Vahtras, Near-edge core photoabsorption in polyenes, J. Chem. Phys. 102 (14) (1995) 5589-5597, http:// dx.doi.org/10.1063/1.469290.

[50] B. Watts, S. Swaraj, D. Nordlund, J. Lüning, H. Ade, Calibrated nexafs spectra of common conjugated polymers, J. Chem. Phys. 134 (2) (2011) 024702, http://dx.doi.org/10.1063/1.3506636.

[51] M. Gliboff, D. Sulas, D. Nordlund, D.W. deQuilettes, P.D. Nguyen, G.T. Seidler, et al., Direct measurement of acceptor group localization on donor-acceptor polymers using resonant auger spectroscopy, J. Phys. Chem. C 118 (10) (2014) 5570-5578, http://dx.doi.org/10.1021/jp412150j.

[52] H. Ade, Characterization of organic thin films with resonant soft x-ray scattering and reflectivity near the carbon and fluorine absorption edges, Eur. Phys. J. Spec. Top. 208 (1) (2012) 305-318, http://dx.doi.org/10.1140/ epjst/e2012-01626-y.

[53] H. Yan, C. Wang, A.R. McCarn, H. Ade, Accurate and facile determination of the index of refraction of organic thin films near the carbon absorption edge, Phys. Rev. Lett. 110 (2013) 177401, http://dx.doi.org/10.1103/ PhysRevLett.110.177401.

[54] C. Wang, T. Araki, B. Watts, S. Harton, T. Koga, S. Basu, et al., Resonant soft xray reflectivity of organic thin films, J. Vac. Sci. Technol. A 25 (2007) 575-586, http://dx.doi.org/10.1116/1.2731352.

[55] H. Yan, C. Wang, A. Garcia, S. Swaraj, Z. Gu, C.R. McNeill, et al., Interfaces in organic devices studied with resonant soft x-ray reflectivity, J. Appl. Phys. 110 (10) (2011) 102220, http://dx.doi.org/10.1063/1.3661991.

[56] D.F. Sunday, R.J. Kline, Reducing block copolymer interfacial widths through polymer additives, Macromolecules 48 (3) (2015) 679-686, http:// dx.doi.org/10.1021/ma502015u.

[57] C.F. Welch, R.P. Hjelm, J.T. Mang, M.E. Hawley, D.A. Wrobleski, E. Bruce Orler, et al., Resonant soft $\mathrm{x}$-ray scattering and reflectivity study of the phaseseparated structure of thin poly(styrene-b-methyl methacrylate) films, J. Polym. Sci. B Polym. Phys. 51 (2) (2013) 149-157, http://dx.doi.org/ 10.1002/polb.23190.

[58] M. Mezger, B. Jérôme, J.B. Kortright, M. Valvidares, E.M. Gullikson, A. Giglia, et al., Molecular orientation in soft matter thin films studied by resonant soft x-ray reflectivity, Phys. Rev. B 83 (2011) 155406, http://dx.doi.org/10.1103/
PhysRevB.83.155406,

[59] M. Mezger, B.M. Ocko, H. Reichert, M. Deutsch, Surface layering and melting in an ionic liquid studied by resonant soft x-ray reflectivity, Proc. Natl. Acad. Sci. $110 \quad$ (10) (2013) 3733-3737, http://dx.doi.org/10.1073/ pnas. 1211749110.

[60] L. Pasquali, S. Mukherjee, F. Terzi, A. Giglia, N. Mahne, K. Koshmak, et al., Structural and electronic properties of anisotropic ultrathin organic films from dichroic resonant soft x-ray reflectivity, Phys. Rev. B 89 (2014) 045401 http://dx.doi.org/10.1103/PhysRevB.89.045401.

[61] H. Ade, A.P. Hitchcock, Nexafs microscopy and resonant scattering: composition and orientation probed in real and reciprocal space, Polymer 49 (3) (2008) 643-675, http://dx.doi.org/10.1016/j.polymer.2007.10.030.

[62] J. Fink, E. Schierle, E. Weschke, J. Geck, Resonant elastic soft x-ray scattering, Rep. Prog. Phys. 76 (5) (2013) 056502, http://dx.doi.org/10.1088/0034-4885/ 76/5/056502.

[63] J.M. Virgili, Y. Tao, J.B. Kortright, N.P. Balsara, R.A. Segalman, Analysis of order formation in block copolymer thin films using resonant soft x-ray scattering Macromolecules 40 (6) (2007) 2092-2099, http://dx.doi.org/10.1021] ma061734k

[64] C. Wang, D.H. Lee, A. Hexemer, M.I. Kim, W. Zhao, H. Hasegawa, et al. Defining the nanostructured morphology of triblock copolymers using resonant soft x-ray scattering, Nano Lett. 11 (9) (2011) 3906-3911.

[65] F. Liu, M.A. Brady, C. Wang, Resonant soft x-ray scattering for polymer materials, Eur. Polym. J. (2016), http://dx.doi.org/10.1016/ j.eurpolymj.2016.04.014.

[66] A. Hexemer, P. Müller-Buschbaum, Advanced grazing-incidence techniques for modern soft-matter materials analysis, IUCrJ 2 (2015) 106-125, http:// dx.doi.org/10.1107/S2052252514024178.

[67] H.F. Gleeson, L.S. Hirst, Resonant x-ray scattering: a tool for structure elucidation in liquid crystals, Chem. Phys. Chem. 7 (2006) 321-328, http:/ dx.doi.org/10.1002/cphc.200500426.

[68] B.A. Collins, J.E. Cochran, H. Yan, E. Gann, C. Hub, R. Fink, et al., Polarized xray scattering reveals non-crystalline orientational ordering in organic films, Nat. Mater. 11 (2012) 536-543, http://dx.doi.org/10.1038/nmat3310.

[69] B.A. Collins, Z. Li, J.R. Tumbleston, E. Gann, C.R. McNeill, H. Ade, Absolute measurement of domain composition and nanoscale size distribution explains performance in PTB7:PC71BM solar cells, Adv. Energy Mater. 3 (1) (2013) 65-74, http://dx.doi.org/10.1002/aenm.201200377.

[70] W. Ma, J.R. Tumbleston, M. Wang, E. Gann, F. Huang, H. Ade, Domain purity, miscibility, and molecular orientation at donor/acceptor interfaces in high performance organic solar cells: paths to further improvement, Adv. Energy Mater 3 (7) (2013) 864-872, http://dx.doi.org/10.1002/aenm.201200912.

[71] J.R. Tumbleston, B.A. Collins, L. Yang, A.C. Stuart, E. Gann, W. Ma, et al., The influence of molecular orientation on organic bulk heterojunction solar cells, Nat. Photonics 8 (2014) 385-391, http://dx.doi.org/10.1038/ nphoton.2014.55.

[72] Y. Diao, Y. Zhou, T. Kurosawa, L. Shaw, C. Wang, S. Park, et al., Flow-enhanced solution printing of all-polymer solar cells, Nat. Commun. 6 (2015) 7955 http://dx.doi.org/10.1038/ncomms8955.

[73] T. Araki, H. Ade, J.M. Stubbs, D.C. Sundberg, G.E. Mitchell, J.B. Kortright, et al., Resonant soft x-ray scattering from structured polymer nanoparticles, Appl. Phys. Lett. 89 (12) (2006) 124106, http://dx.doi.org/10.1063/1.2356306.

[74] D.T. Wong, C. Wang, K.M. Beers, J.B. Kortright, N.P. Balsara, Mesoporous block copolymer morphology studied by contrast-matched resonant soft x-ray scattering, Macromolecules 45 (22) (2012) 9188-9195, http://dx.doi.org/ 10.1021/ma3019206.

[75] E. Gann, B.A. Collins, M. Tang, J.R. Tumbleston, S. Mukherjee, H. Ade, Origins of polarization-dependent anisotropic x-ray scattering from organic thin films, J. Synchrotron Rad. 23 (1) (2016) 219-227, http://dx.doi.org/10.1107| S1600577515019074.

[76] F. Liu, C. Wang, J.K. Baral, L. Zhang, J.J. Watkins, A.L. Briseno, et al., Relating chemical structure to device performance via morphology control in diketopyrrolopyrrole-based low band gap polymers, J. Am. Chem. Soc. 135 (51) (2013) 19248-19259, http://dx.doi.org/10.1021/ja408923y.

[77] K.H. Stone, J.B. Kortright, Molecular anisotropy effects in carbon $k$-edge scattering: depolarized diffuse scattering and optical anisotropy, Phys. Rev. B 90 (2014) 104201, http://dx.doi.org/10.1103/PhysRevB.90.104201.

[78] C. Zhu, C. Wang, A. Young, F. Liu, I. Gunkel, D. Chen, et al., Probing and controlling liquid crystal helical nanofilaments, Nano Lett. 15 (5) (2015) 3420-3424, http://dx.doi.org/10.1021/acs.nanolett.5b00760.

[79] C. Zhu, M.R. Tuchband, A. Young, M. Shuai, A. Scarbrough, D.M. Walba, et al. Resonant carbon $k$-edge soft $\mathrm{x}$-ray scattering from lattice-free heliconical molecular ordering: soft dilative elasticity of the twist-bend liquid crystal phase, Phys. Rev. Lett. 116 (2016) 147803, http://dx.doi.org/10.1103/ PhysRevLett.116.147803.

[80] M. Kiguchi, G. Yoshikawa, K. Saiki, Temperature and thickness dependence of molecular orientation of $\alpha$-sexithienyl on Cu(111), J. Appl. Phys. 94 (8) (2003) 4866-4870, http://dx.doi.org/10.1063/1.1609637.

[81] M. Kiguchi, S. Entani, K. Saiki, G. Yoshikawa, One-dimensional ordered structure of $\alpha$-sexithienyl on $\mathrm{Cu}(110)$ ). Appl. Phys. Lett. 84 (18) (2004) 3444-3446, http://dx.doi.org/10.1063/1.1736315.

[82] G. Yoshikawa, M. Kiguchi, S. Ikeda, K. Saiki, Molecular orientations and adsorption structures of $\alpha$-sexithienyl thin films grown on $\operatorname{Ag}(110)$ and Ag(111) surfaces, Surf. Sci. 559 (2004) 77-84, http://dx.doi.org/10.1016/ j.susc.2004.04.045. 
[83] R. Onoki, G. Yoshikawa, Y. Tsuruma, S. Ikeda, K. Saiki, K. Ueno, Nanotransfer of the polythiophene molecular alignment onto the step-bunched vicina Si(111) substrate, Langmuir 24 (20) (2008) 11605-11610, http://dx.doi.org/ 10.1021/la8016722.

[84] U. Aygül, D. Batchelor, U. Dettinger, S. Yilmaz, S. Allard, U. Scherf, et al. Molecular orientation in polymer films for organic solar cells studied by nexafs, J. Phys. Chem. C 116 (7) (2012) 4870-4874, http://dx.doi.org/ 10.1021 /jp205653n.

[85] H. Ikeura-Sekiguchi, T. Sekiguchi, Molecular ordering effect of regioregular poly(3-hexylthiophene) using sulfur k-edge x-ray absorption spectroscopy Jpn. J. Appl. Phys. 53 (2014) 02BB07, http://dx.doi.org/10.7567 JUAP.53.02BB07.

[86] D. Batchelor, U. Aygül, U. Dettinger, M. Ivanovic, A. Tournebize, S. Mangold, et al., Insight into the orientation of LBG polymer films by XANES experiment and calculation, Eur. Polym. J. (2016), http://dx.doi.org/10.1016/ j.eurpolymj.2016.04.005.

[87] C. Arantes, B.G.A.L. Borges, B. Beck, G. Araújo, L.S. Roman, M.L.M. Rocco, Femtosecond electron delocalization in poly(thiophene) probed by resonant auger spectroscopy, J. Phys. Chem. C 117 (16) (2013) 8208-8213, http:/| dx.doi.org/10.1021/jp312660d.

[88] A.P. Hitchcock, J.A. Horsley, J. Stöhr, Inner shell excitation of thiophene and thiolane: gas, solid, and monolayer states, J. Chem. Phys. 85 (9) (1986) 4835-4848, http://dx.doi.org/10.1063/1.451718.

[89] T. Skotheim, X. Yang, J. Chen, P. Hale, T. Inagaki, L. Samuelson, et al., Highly ordered thin films of polyheterocycles: a synchrotron radiation study of polypyrrole and polythiophene Langmuir-Blodgett films, Synt. Met. 28 (1) (1989) 229-236, http://dx.doi.org/10.1016/0379-6779(89)90526-2.

[90] T. Fransson, D. Burdakova, P. Norman, K- and l-edge X-ray absorption spectrum calculations of closed-shell carbon, silicon, germanium, and sulfur compounds using damped four-component density functional response theory, Phys. Chem. Chem. Phys. 18 (2016) 13591-13603, http://dx.doi.org/ 10.1039/C6CP00561F.

[91] P. Mach, R. Pindak, A.M. Levelut, P. Barois, H.T. Nguyen, H. Baltes, et al., Structures of chiral smectic- $C$ mesophases revealed by polarizationanalyzed resonant X-ray scattering, Phys. Rev. E 60 (1999) 6793-6802 http://dx.doi.org/10.1103/PhysRevE.60.6793.

[92] A. Cady, J.A. Pitney, R. Pindak, L.S. Matkin, S.J. Watson, H.F. Gleeson, et al Orientational ordering in the chiral smectic- $\mathrm{c}_{\mathrm{fi} 2}^{*}$ liquid crystal phase determined by resonant polarized x-ray diffraction, Phys. Rev. E 64 (2001) 050702, http://dx.doi.org/10.1103/PhysRevE.64.050702.

[93] C.L. Folcia, J. Ortega, J. Etxebarria, S. Rodriguez-Conde, G. Sanz-Enguita, K. Geese, et al., Spontaneous and field-induced mesomorphism of a silylterminated bent-core liquid crystal as determined from second-harmonic generation and resonant X-ray scattering, Soft Matter. 10 (2014) 196-205, http://dx.doi.org/10.1039/C3SM51277K.

[94] J. Mårdalen, C. Riekel, H. Müller, Anomalous X-ray scattering at the sulfur edge of poly(3-octylthiophene), J. Appl. Cryst. 27 (2) (1994) 192-195, http:/ dx.doi.org/10.1107/S0021889893009835.

[95] S.T. Chourou, A. Sarje, X.S. Li, E.R. Chan, A. Hexemer, HipGISAXS: a highperformance computing code for simulating grazing-incidence $\mathrm{X}$-ray scattering data, J. Appl. Cryst. 46 (6) (2013) 1781-1795, http://dx.doi.org/ $10.1107 /$ S0021889813025843.

[96] G. Renaud, R. Lazzari, F. Leroy, Probing surface and interface morphology with grazing incidence small angle x-ray scattering, Surf. Sci. Rep. 64 (8) (2009) 255-380, http://dx.doi.org/10.1016/j.surfrep.2009.07.002.

[97] Z. Jiang, D.R. Lee, S. Narayanan, J. Wang, S.K. Sinha, Waveguide-enhanced grazing-incidence small-angle $\mathrm{x}$-ray scattering of buried nanostructures in thin films, Phys. Rev. B 84 (2011) 075440, http://dx.doi.org/10.1103/ PhysRevB.84.075440.

[98] D.F. Sunday, M.R. Hammond, C. Wang, W. li Wu, D.M. Delongchamp, M. Tjio et al., Determination of the internal morphology of nanostructures patterned by directed self assembly, ACS Nano 8 (8) (2014) 8426-8437.

[99] D.F. Sunday, S. List, J.S. Chawla, R.J. Kline, Evaluation of the effect of data quality on the profile uncertainty of critical dimension small angle x-ray scattering, J. Micro/Nanolith 15 (2016) 014001, http://dx.doi.org/10.1117/ 1.JMM.15.1.014001.

[100] K.W. Chou, B. Yan, R. Li, E.Q. Li, K. Zhao, D.H. Anjum, et al., Spin-cast bulk heterojunction solar cells: a dynamical investigation, Adv. Mater. 25 (13) (2013) 1923-1929, http://dx.doi.org/10.1002/adma.201203440.

[101] X. Gu, I. Gunkel, A. Hexemer, W. Gu, T.P. Russell, An in situ grazing incidence $\mathrm{x}$-ray scattering study of block copolymer thin films during solvent vapor annealing, Adv. Mater. 26 (2) (2014) 273-281, http://dx.doi.org/10.1002/ adma.201302562.

[102] L.J. Richter, D.M. DeLongchamp, F.A. Bokel, S. Engmann, K.W. Chou, A. Amassian, et al., In situ morphology studies of the mechanism for solution additive effects on the formation of bulk heterojunction films, Adv. Energy Mater 5 (3) (2015) 1400975, http://dx.doi.org/10.1002/aenm.201400975.

[103] S. Pröller, F. Liu, C. Zhu, C. Wang, T.P. Russell, A. Hexemer, et al., Following the morphology formation in situ in printed active layers for organic solar cells, Adv. Energy Mater 6 (1) (2016) 1501580, http://dx.doi.org/10.1002/ aenm.201501580.

[104] A. Sarje, X.S. Li, S. Chourou, E.R. Chan, A. Hexemer, Massively parallel x-ray scattering simulations, High Perform. Comput. Netw. Storage Anal. (SC) 2012 (2012) 1-11, http://dx.doi.org/10.1109/SC.2012.76. International Conference for.
[105] A. Sarje, X.S. Li, A. Hexemer, High Performance Computing Systems. Performance Modeling, Benchmarking and Simulation, Springer International Publishing, 2014

[106] T.M. McDonald, J.A. Mason, X. Kong, E.D. Bloch, D. Gygi, A. Dani, et al., Cooperative insertion of $\mathrm{CO}_{2}$ in diamine-appended metal-organic frameworks, Nature 519 (2015) 303-308, http://dx.doi.org/10.1038/nature14327.

[107] W.S. Drisdell, R. Poloni, T.M. McDonald, T.A. Pascal, L.F. Wan, C.D. Pemmaraju, et al., Probing the mechanism of $\mathrm{CO}_{2}$ capture in diamineappended metal-organic frameworks using measured and simulated x-ray spectroscopy, Phys. Chem. Chem. Phys. 17 (2015) 21448-21457, http:// dx.doi.org/10.1039/c5cp02951a.

[108] E. Helfand, Y. Tagami, Theory of the interface between immiscible polymers, J. Polym. Sci. B Polym. Phys. 9 (10) (1971) 741-746, http://dx.doi.org/ 10.1002/pol.1971.110091006.

[109] E. Helfand, Y. Tagami, Theory of the interface between immiscible polymers, Ii. J. Chem. Phys. 56 (7) (1972) 3592-3601, http://dx.doi.org/10.1063/ 1.1677735 .

[110] D. Broseta, G.H. Fredrickson, E. Helfand, L. Leibler, Molecular weight and polydispersity effects at polymer-polymer interfaces, Macromolecules 23 (1) (1990) 132-139, http://dx.doi.org/10.1021/ma00203a023.

[111] R.J. Roe, Methods of X-ray and Neutron Scattering in Polymer Science, Oxford University Press, 2000.

[112] R.L. Leheny, Xpcs: nanoscale motion and rheology, Curr. Opin. Colloid Interface Sci. 17 (1) (2012) 3-12, http://dx.doi.org/10.1016/ j.cocis.2011.11.002.

[113] G. Grubel, F. Zontone, Correlation spectroscopy with coherent x-rays, J. Alloys Compd. 362 (1-2) (2004) 3-11, http://dx.doi.org/10.1016/S09258388(03)00555-3.

[114] F. Livet, Diffraction with a coherent X-ray beam: dynamics and imaging, Acta Crystallogr. Sect. A Found. Crystallogr. 63 (2007) 87-107, http://dx.doi.org/ 10.1107/S010876730605570x.

[115] M. Sutton, A review of x-ray intensity fluctuation spectroscopy, Comptes Rendus Phys. 9 (5-6) (2008) 657-667, http://dx.doi.org/10.1016/ j.crhy.2007.04.008.

[116] L. Cristofolini, Synchrotron x-ray techniques for the investigation of structures and dynamics in interfacial systems, Curr. Opin. Colloid Interface Sci. 19 (3) (2014) 228-241, http://dx.doi.org/10.1016/j.cocis.2014.03.006.

[117] E. Gulari, E. Gulari, Y. Tsunashima, B. Chu, Polymer diffusion in a dilute theta solution .1. polystyrene in cyclohexane, Polymer 20 (3) (1979) 347-355, http://dx.doi.org/10.1016/0032-3861(79)90099-5.

[118] S.C. Jung, Y.C. Bae, The effects of interaction energy on the volume phase transition of n-isopropylacrylamide-co-n-isopropylmethacrylamide nanosized gel particles: applicability of molecular simulation technique, Polymer 50 (20) (2009) 4957-4963, http://dx.doi.org/10.1016/ j.polymer.2009.08.006.

[119] S.C. Jung, S.Y. Oh, Y.C. Bae, Reentrant swelling behavior of thermosensitive nisopropylacrylamide nano-sized gel particles, Polymer 50 (14) (2009) 3370-3377, http://dx.doi.org/10.1016/j-polymer 2009.05.011.

[120] S.M. Kim, Y.C. Bae, Co-nonsolvency effect of thermosensitive n-isopropylacrylamide nanometer-sized gel particles in water-peg systems, Polymer 54 (8) (2013) 2138-2145, http://dx.doi.org/10.1016/ j.polymer.2013.02.014.

[121] S.M. Lee, Y.C. Bae, Enhanced solvation effect of re-collapsing behavior for cross-linked pmma particle gel in aqueous alcohol solutions, Polymer 55 (18) (2014) 4684-4692, http://dx.doi.org/10.1016/j.polymer.2014.07.033.

[122] S.Y. Oh, H.J. Kim, Y.C. Bae, Molecular thermodynamic analysis for phase transitions of linear and cross-linked poly(n-isopropylacrylamide) in water/ 2-propanol mixtures, Polymer 54 (25) (2013) 6776-6784, http://dx.doi.org/ 10.1016/j.polymer.2013.10.026.

[123] M. Sedlak, C. Konak, P. Stepanek, J. Jakes, Semidilute solutions of poly(methacrylic acid) in the absence of salt - dynamic light-scattering study, Polymer 28 (6) (1987) 873-880, http://dx.doi.org/10.1016/0032-3861(87) 90156-X.

[124] H.I. Tang, P.L. Johnson, E. Gulari, Styrene polymerized in an oil-in-water microemulsion, Polymer 25 (9) (1984) 1357-1362, http://dx.doi.org/ 10.1016/0032-3861(84)90391-4.

[125] F.P. Yi, S.X. Zheng, Effect of hydrophobic polystyrene microphases on temperature-responsive behavior of poly(n-isopropylacrylamide) hydrogels, Polymer 50 (2) (2009) 670-678, http://dx.doi.org/10.1016/ j.polymer.2008.11.038.

[126] P. Gupta, C. Elkins, T.E. Long, G.L. Wilkes, Electrospinning of linear homopolymers of poly(methyl methacrylate): exploring relationships between fiber formation, viscosity, molecular weight and concentration in a good solvent, Polymer 46 (13) (2005) 4799-4810, http://dx.doi.org/10.1016/ j.polymer.2005.04.021.

[127] K. Kita-Tokarczyk, J. Grumelard, T. Haefele, W. Meier, Block copolymer vesicles - using concepts from polymer chemistry to mimic biomembranes, Polymer 46 (11) (2005) 3540-3563, http://dx.doi.org/10.1016/ j.polymer.2005.02.083.

[128] S.K. Sinha, Z. Jiang, L.B. Lurio, X-ray photon correlation spectroscopy studies of surfaces and thin films, Adv. Mater. 26 (46) (2014) 7764-7785, http:// dx.doi.org/10.1002/adma.201401094.

[129] J. Jackle, The spectrum of surface waves on viscoelastic liquids of arbitrary depth, J. Phys. Condens Matter. 10 (32) (1998) 7121-7131, http://dx.doi.org/ 10.1088/0953-8984/10/32/004. 
[130] T. Hoshino, M. Kikuchi, D. Murakami, Y. Harada, K. Mitamura, K. Ito, et al., Xray photon correlation spectroscopy using a fast pixel array detector with a grid mask resolution enhancer, J. Synchrotron Rad. 19 (2012) 988-993, http://dx.doi.org/10.1107/S0909049512038769.

[131] B. Frieberg, J. Kim, S. Narayanan, P.F. Green, Surface layer dynamics in miscible polymer blends, Acs Macro Lett. 2 (5) (2013) 388-392, http:// dx.doi.org/10.1021/mz400104p.

[132] B. Frieberg, J. Kim, S. Narayanan, P.F. Green, Surface dynamics of miscible polymer blend nanocomposites, Acs Nano 8 (1) (2014) 607-613, http:/ dx.doi.org/10.1021/nn405233a.

[133] F. Livet, F. Bley, F. Ehrburger-Dolle, I. Morfin, E. Geissler, M. Sutton, Homodyne and heterodyne $\mathrm{x}$-ray photon correlation spectroscopy: latex particles and elastomers, J. Appl. Crystallogr. 40 (2007) S38-S42, http://dx.doi.org/ $10.1107 /$ S0021889807003561.

[134] F. Livet, F. Bley, F. Ehrburger-Dolle, I. Morfin, E. Geissler, M. Sutton, X-ray intensity fluctuation spectroscopy by heterodyne detection, J Synchrotron Rad 13 (2006) 453-458, http://dx.doi.org/10.1107/S0909049506030044.

[135] C. Steier, et al., Proposal for a soft x-ray diffraction limited upgrade of the ALS, in: Proceedings, 5th International Particle Accelerator Conference (IPAC 2014), 2014 p. MOPME084.

[136] A. Ricci, Nanoscale dynamics in complex materials by resonant x-ray photon correlation spectroscopy (rxpcs), J. Supercond. Nov. Magn. 28 (4) (2015) 1295-1298, http://dx.doi.org/10.1007/s10948-014-2907-3.

[137] S. Konings, C. Schussler-Langeheine, H. Ott, E. Weschke, E. Schierle, H. Zabel, et al., Magnetic domain fluctuations in an antiferromagnetic film observed with coherent resonant soft x-ray scattering, Phys. Rev. Lett. 106 (7) (2011), http://dx.doi.org/10.1103/PhysRevLett.106.077402.

[138] Z. Bao, R. Springell, H.C. Walker, H. Leiste, K. Kuebel, R. Prang, et al., Antiferromagnetism in uo2 thin epitaxial films, Phys. Rev. B 88 (13) (2013), http://dx.doi.org/10.1103/PhysRevB.88.134426.

[139] S.W. Chen, H. Guo, K.A. Seu, K. Dumesnil, S. Roy, S.K. Sinha, Jamming behavior of domains in a spiral antiferromagnetic system, Phys. Rev. Lett. 110 (21) (2013), http://dx.doi.org/10.1103/PhysRevLett.110.217201.

[140] T. Matsumura, H. Nakao, Y. Murakami, Resonant x-ray scattering experiments on the ordering of electronic degrees of freedom, J. Phys. Soc. Jpn. 82 (2) (2013), http://dx.doi.org/10.7566/Jpsj.82.021007.

[141] M. Ewerlin, B. Pfau, C.M. Gunther, S. Schaffert, S. Eisebitt, R. Abrudan, et al. Exploration of magnetic fluctuations in pdfe films, J. Phys. Condens Matter 25 (26) (2013), http://dx.doi.org/10.1088/0953-8984/25/26/266001.

[142] A.G. McDermott, P.M. Budd, N.B. McKeown, C.M. Colina, J. Runt, Physical aging of polymers of intrinsic microporosity: a saxs/waxs study, J. Mater. Chem. A 2 (2014) 11742-11752, http://dx.doi.org/10.1039/C4TA02165G.

[143] X.C. Chen, J.B. Kortright, N.P. Balsara, Water uptake and proton conductivity in porous block copolymer electrolyte membranes, Macromolecules 48 (16) (2015) 5648-5655, http://dx.doi.org/10.1021/acs.macromol.5b00950.

[144] D.T. Wong, C. Wang, J.A. Pople, N.P. Balsara, Effect of nonsolvent exposure on morphology of mesoporous semicrystalline block copolymer films, Macromolecules 46 (11) (2013) 4411-4417, http://dx.doi.org/10.1021/ ma400051x.

[145] I. Gurevitch, R. Buonsanti, A.A. Teran, B. Gludovatz, R.O. Ritchie, J. Cabana, e al., Nanocomposites of titanium dioxide and polystyrene-poly(ethylene oxide) block copolymer as solid-state electrolytes for lithium metal batteries, J. Electrochem Soc. 160 (9) (2013) A1611-A1617, http://dx.doi.org/10.1149/ 2.117309jes.

[146] K. Schmidt-Rohr, Q Chen, Parallel cylindrical water nanochannels in nafion fuel-cell membranes, Nat. Mater. 7 (2008) 75-83, http://dx.doi.org/10.1038/ nmat2074.

[147] M.A. Modestino, A. Kusoglu, A. Hexemer, A.Z. Weber, R.A. Segalman, Controlling nafion structure and properties via wetting interactions, Macromolecules 45 (11) (2012) 4681-4688, http://dx.doi.org/10.1021/ma300212f.

[148] A. Kusoglu, M.A. Modestino, A. Hexemer, R.A. Segalman, A.Z. Weber, Subsecond morphological changes in nafion during water uptake detected by small-angle x-ray scattering, ACS Macro Lett. 1 (1) (2012) 33-36, http:// dx.doi.org/10.1021/mz200015c

[149] K. Kanai, Y. Noda, K. Kato, T. Kubo, K. Iketaki, A. Shimizu, et al., Electronic structure of delocalized singlet biradical $\mathrm{Ph}_{2}$-IDPL solid film, Phys. Chem. Chem. Phys. 12 (2010) 12570-12577, http://dx.doi.org/10.1039/c0cp00178c.

[150] D.T. Duong, H. Phan, D. Hanifi, P.S. Jo, T.Q. Nguyen, A. Salleo, Direct observation of doping sites in temperature-controlled, p-doped P3HT thin films by conducting atomic force microscopy, Adv. Mater. 26 (35) (2014) 6069-6073, http://dx.doi.org/10.1002/adma.201402015.

151] H.C. Tian, J.Q. Liu, D.X. Wei, X.Y. Kang, C. Zhang, J.C. Du, et al., Graphene oxide doped conducting polymer nanocomposite film for electrode-tissue interface, Biomaterials 35 (7) (2014) 2120-2129, http://dx.doi.org/10.1016/ j.biomaterials.2013.11.058.

[152] M. Magnuson, J.H. Guo, S.M. Butorin, A. Agui, C. Såthe, J. Nordgren, et al, The electronic structure of polyaniline and doped phases studied by soft X-ray absorption and emission spectroscopies, J. Chem. Phys. 111 (10) (1999) 4756-4761, http://dx.doi.org/10.1063/1.479238.

[153] D. Guay, G. Tourillon, A. Fontaine, Electrochemical inclusion of copper and iron species in a conducting polymer observed in situ using time-resolved $\mathrm{x}$ ray absorption spectroscopy, Faraday Discuss. Chem. Soc. 89 (1990) 41-50, http://dx.doi.org/10.1039/DC9908900041.

[154] E. Aziz, A. Vollmer, S. Eisebitt, W. Eberhardt, P. Pingel, D. Neher, et al, Localized charge transfer in a molecularly doped conducting polymer, Adv. Mater. 19 (20) (2007) 3257-3260, http://dx.doi.org/10.1002/ adma.200700926.

[155] J.E. Cochran, M.J.N. Junk, A.M. Glaudell, P.L. Miller, J.S. Cowart, M.F. Toney, et al., Molecular interactions and ordering in electrically doped polymers: blends of PBTTT and F4TCNQ, Macromolecules 47 (19) (2014) 6836-6846, http://dx.doi.org/10.1021/ma501547h. 